\title{
Capacity Optimization under Uncertainty: The Impact of Operational Time Lags
}

\author{
Hettie J. Boonman
}

TNO, The Netherlands Organization for Applied Scientific Research, The Netherlands

Afzal S. Siddiqui*

Department of Statistical Science, University College London, UK, Department of Computer and Systems Sciences, Stockholm University, Sweden, and Department of Decision Sciences, HEC Montréal, Canada

\begin{abstract}
Time lags in switching operational modes are typical in the manufacturing and power sectors but are not treated in most real options models. In this paper, we consider a firm that has the opportunity to suspend and to resume production infinitely many times subject to a time lag after each startup decision. We contribute to the literature by allowing the firm to determine its level of installed capacity in conjunction with its optimal investment timing. We find that an increase in the length of the time lag results in an increase in the optimal capacity level. Capacity optimization also interacts with the length of the time lag to affect investment timing and the triggers to suspend and resume production, thereby weakening the result about hysteresis from a standard real options model. Under the assumption of a fixed level of capacity, a longer lag speeds up the decision to resume operations due to a positive upside to the revenue but delays the suspension of operations. By contrast, with capacity optimization, a longer time lag results in a larger capacity choice, which can indirectly delay the investment decision and the timing to resume operations. This indirect effect dominates when the level of market uncertainty is low and the time lag is initially small.
\end{abstract}

Keywords: Investment Analysis, Real Options, Time Lag, Capacity Optimization

\section{Introduction}

An advantage of the real options approach (Dixit and Pindyck, 1994) over now-or-never net present value (NPV) appraisal methods is its capability to account for the managerial discretion to modify the project after the initial investment decision. Indeed, such so-called embedded options can have a bearing upon the initial investment decision and the value

\footnotetext{
${ }^{*}$ Corresponding author

Email address: afzal.siddiqui@ucl.ac.uk (Afzal S. Siddiqui*)
} 
of the option to invest itself. With the recession that started in the fall of 2008, valuing firms' flexibility to respond to market conditions is paramount. For example, in December 2008, General Motors announced that it would "temporarily close twenty factories across North America and make sweeping cuts to its vehicle production as it tries to adjust to dramatically weaker automobile demand."'1 Other car manufacturers, such as Toyota2 and Honda ${ }^{3}$ similarly chose to close some of their production plants temporarily. In turn, the global steel market, which experienced a major cutback in steel purchases from its customers, i.e., automotive manufacturers, was also affected by the global economic downturn. To illustrate, in May 2009, the world's largest steel producer, ArcelorMittal, opted to idle its Monessen coke plant $4^{4}$ In subsequent years, steel makers recovered from the slump that had begun when the economy faltered in 2008. Eventually, in 2012, ArcelorMittal announced the resumption of production at its Monessen coke plant. Yet, it took two years for production to resume. In general, such production facilities are characterized by time lags to resume production from a suspended state. Moreover, there are fixed costs associated with switching between the operational and the suspended state.

Given this background, we assess the decision-making problem of a firm with the opportunity to suspend and to resume production infinitely many times in the future in exchange for some positive switching costs. Entry-and-exit models have been pioneered by Mossin (1968) and generalized by Brennan and Schwartz (1985), Dixit (1989), and McDonald (2002). Where these models place their focus on the optimal triggers for switching operational states, some later extensions take up the additional challenge to optimize endogenously the firm's capacity. For example, Van Mieghem and Dada (1999) consider a firm that optimizes the capacity of its plant before demand uncertainty has been resolved. After demand realization, the firm chooses optimal production quantities, which are constrained by the earlier chosen capacity size. Dangl (1999) and Hagspiel et al. (2016) extend this model to a dynamic setting in which the firm optimizes investment timing along with capacity size. After investment, the firm optimizes the level of production for each realization of demand assuming the same constraints as in Van Mieghem and Dada (1999). Likewise, Boonman et al. (2015) examine a strategic capacity investment game between two firms.

Such models provide a contribution to the existing literature by optimizing the level of capacity. However, they typically allow a firm to switch costlessly between the operational

\footnotetext{
${ }^{1}$ http://articles.economictimes.indiatimes.com/2008-12-13/news/28479230_1_tony-sapienza-gm-andchrysler-vehicle-production

${ }^{2}$ http://content.time.com/time/business/article/0,8599,1919395,00.html

${ }^{3}$ http://www.theguardian.com/business/2009/jan/30/honda-swindon-shutdown

${ }^{4}$ http://www.post-gazette.com/business/2014/04/08/Monessen-coke-plant-set-to-resume-productionthis-month/stories/201404080025
} 
and idle states. In Dixit (1989) and McDonald (2002), a fixed cost has to be paid for switching between these two states, which brings the model closer to reality. Indeed, laying off employees or depreciation of unused equipment results in non-negligible costs when a firm decides to suspend operations. Furthermore, resuming production generates costs like overdue maintenance, marketing, and training that comes with hiring new employees. In the case of the steel producer example, ArcelorMittal even re-invested $\$ 50$ million in its plant prior to the re-opening.

In this paper, we incorporate such realistic features into a real options model for capacity sizing and investment timing in order to gain deeper managerial insights about the impact of time lags and capacity sizing on hysteresis in a firm's operations. Specifically, the standard real options model of industry entry and exit by a firm under market uncertainty assumes a fixed capacity and instantaneous suspension/resumption possibilities (Dixit and Pindyck. 1994). Consequently, greater uncertainty increases (decreases) the entry (exit) price threshold. In effect, more volatility causes the firm to delay making decisions of either sort. Since many firms, e.g., ArcelorMittal as we describe, have production facilities that require lead times before being re-activated from idle states and also have the flexibility to choose their capacity size at the time of investment, we extend the real options approach to examine how these two key features affect firm behavior.

We address the issue of time lags by introducing a delay in switching between idle and operational states, which takes place after the decision to resume production. Even for modest time lags, there is a significant effect on the optimal triggers to switch from the operational to the idle state and vice versa. In related work, Bar-Ilan and Strange (1996) embed lags in the classic irreversible lumpy investment model presented by Dixit (1989). They find that for some parameter values, an increase in uncertainty can actually hasten investment, a result contrary to that found in papers without investment lags. Meanwhile, Takashima et al. (2010) report a similar finding in examining investment timing and discrete capacity choice when there is a time lag between the initial investment decision and the start of operations. Other work on time lags typically omits the capacity-sizing decision, e.g., Gauthier and Morellec (2000) and Costeniuc et al. (2008). The former investigates the timing of uncertain investment decisions given an implementation delay after the investment decision without either capacity sizing or operational flexibility. Costeniuc et al. (2008) model a firm that can make investment and disinvestment decisions with time lags but without capacity sizing. Furthermore, the time lags are not after the investment decision. Instead, a Parisian delay is assumed, i.e., a Parisian option gets activated/deactivated when the underlying process has spent a sufficient amount of time above/below the barrier level.

We incorporate a time lag in McDonald (2002)'s entry-and-exit model not only after the 
entry decision (Bar-Ilan and Strange, 1996; Takashima et al., 2010) but also after each decision to resume production since a startup will not occur instantaneously, a feature that is ignored in capacity-sizing real options work (Dangl, 1999). While the time it takes to prepare the production process is captured by this time lag, the decision to stop the production is assumed to be executed immediately. The paper most closely related to our work is Sødal (2006), who uses the Dixit (1989) model as a baseline and models a firm with infinitely many options to enter and exit a market in exchange for a fixed entry or exit cost. He finds that the effect of a time lag in resuming production implies that increasing uncertainty might hasten investment when there is a time lag and also lower the entry trigger for resuming operations. Our contribution of introducing capacity optimization in the model with operational flexibility and a time lag changes the results of Bar-Ilan and Strange (1996) and Sødal (2006), viz., that the effect of uncertainty on the optimal operational triggers is ambiguous with a fixed capacity level. In fact, with capacity optimization, there is also an indirect effect: greater uncertainty delays investment, which allows for a larger capacity level and, in turn, leads to strictly higher switching triggers. Indeed, operational switching triggers occur around the level of price intercept for which the price is equal to the unit production cost. Thus, this indirect effect of uncertainty on the operational triggers via the capacity size dominates the direct effect (Bar-Ilan and Strange, 1996; Sødal, 2006), thereby causing uncertainty to increase both the resumption and suspension thresholds.

Furthermore, capacity optimization changes the effect of the length of the time lag on the optimal investment and switching triggers. Where Bar-Ilan and Strange (1996) observe that the trigger values decrease for an increase in the length of the time lag, we find that for low uncertainty and a low initial time lag, the triggers can also increase. Indirectly, a longer time lag results in a larger capacity choice, which in turn increases the trigger values. This larger capacity choice is the result of (i) a need to recoup forgone profit during the lag and (ii) a truncated downside of investment due to the presence of a suspension option and unlimited upward potential in the revenue. Thus, at the moment that the firm resumes production after the time lag, it expects the profit to have grown. This result will be amplified under a longer time lag, thereby allowing for a larger capacity choice. Hence, our modeling features enable us to study more realistic settings and provide more subtle insights than those from the extant literature, which could be used by policymakers in reforming industrial strategy.

This paper is structured as follows. Modeling assumptions are presented in Section 2. Section 3 analyzes the benchmark model with investment timing, capacity sizing, and operational flexibility without a time lag. In Section 4, a time lag after the decision to resume production is added to the model. Section 5 compares the numerical results for the models with and without a time lag, and Section 6 concludes. All proofs and lengthy derivations are 
in Appendix B and Appendix C.

\section{Modeling Assumptions}

We take the perspective of a firm that has to decide about capacity investment. The decision variables are (i) investment timing, (ii) capacity sizing, and (iii) suspension/resumption timing (see Figure 1) $:^{5}$ After investment and determination of the capacity size, the firm commences operations and earns a profit flow. In exchange for a switching cost, $S>0$, it can decide to stop operations temporarily. In this state, the profit flow of the firm is zero. An inactive firm has the option to re-start production for a switching cost $R>04^{6}$ Due to the (positive) switching costs, a firm will not switch states at the price where its profit is equal to zero. Instead, it switches from the suspended state to the operational state and vice versa at some optimal switching triggers.

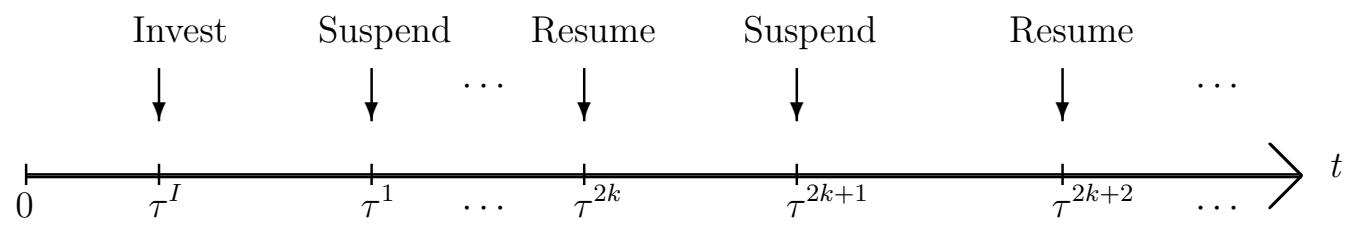

Figure 1: Decision-making timeline for investing, suspending, and resuming operations.

We assume that the inverse demand function at time $t \geq 0$ reflects the price earned by the firm per unit sold and is given by:

$$
P_{t}=X_{t}-\eta Q
$$

where $Q$ is a decision variable representing the fixed annual production of the firm and $\eta \in(0,1)$ is a parameter reflecting the slope of the inverse demand (also known as the substitutability parameter and related to the price elasticity of demand). The parameter $X_{t}$ for $t \geq 0$ is a stochastic shock to the inverse demand outside the control of the decision maker

\footnotetext{
${ }^{5}$ As is typical in real options, each of the timing decisions corresponds to an optimal threshold, i.e., a trigger policy. Thus, when determining the optimal investment threshold, it is necessary to know the optimal capacity size. In turn, prior to determining the optimal capacity size, we need to derive the optimal operational policy. Hence, although the sequence of decisions encountered in real time is $(i) \rightarrow(i i) \rightarrow(i i i)$, since the problem is solved via backward induction, the way that the solution procedure works is $(\mathrm{iii}) \rightarrow(\mathrm{ii}) \rightarrow(\mathrm{i})$.

${ }^{6}$ Positive switching costs are not necessary as long as $R+S>0$. Indeed, either $R$ or $S$ may be negative, which corresponds to a subsidy or a salvage value. However, as described in Chapter 7 of Dixit and Pindyck (1994), if we have $R+S<0$, then it is possible for the firm to make arbitrarily large amounts of money simply by repeatedly suspending and resuming operations.
} 
and is assumed to follow a geometric Brownian motion (GBM):

$$
d X_{t}=\mu X_{t} d t+\sigma X_{t} d \omega_{t}
$$

with drift parameter $\mu$, volatility parameter $\sigma \geq 0, X_{0}=x$, and $d \omega_{t}$ the increment of a standard Wiener process. Future revenues will be discounted at an exogenous interest rate $\rho>\mu$. Variable production cost is denoted by $c \geq 0$, so that the profit flow of a firm in production is given by:

$$
\pi_{t}=\left(P_{t}-c\right) Q
$$

For analytical convenience, we invoke the market-clearing assumption, i.e., the firm produces up to capacity. This assumption is widely used in the literature (Chod and Rudi, 2005, Deneckere et al., 1997; Anand and Girotra, 2007). Unlike Dangl (1999) and Hagspiel et al. (2016), we do not optimize a firm's production level; instead, the firm is able to deal with low prices via the option to suspend the production facility.

Following Dangl (1999) and Hagspiel et al. (2016), investment costs are sunk and equal to $I\left(Q_{t}\right)=\delta Q_{t}^{\lambda}$. Constant $\delta>0$ denotes the variable investment cost, and constant $0<\lambda<1$ indicates a concave investment structure. By adopting the same investment costs, we are able to compare our results with those in the literature $]^{7}$

\section{Operational Flexibility and Capacity Choice without Time Lags}

To illustrate a sequence of decisions made by a firm after investment, consider Figure 2 , which illustrates a sample path of the uncertainty parameter $X_{t}$. Assume that the firm is not producing at the start of the time frame. We find that the firm resumes production at time $t=\tau^{2 k}$ for $k \in \mathbb{N}$. The firm stays in production until it suspends production at time $t=\tau^{2 k+1}$. After this closure decision, the firm resumes production at time $t=\tau^{2 k+2}$. Thus, as part of its optimal investment timing and capacity sizing decisions in Figure 1, the firm must determine the corresponding thresholds of $X_{t}$ at which to suspend and resume operations.

\subsection{The Model}

Figure 1 shows the decision-making timeline. At time $t=\tau^{I}$, the firm invests in optimal capacity size $Q^{*}$. The firm suspends operations at the odd triggers and resumes at the even

[Hagspiel et al. (2016) additionally show a scenario in which a firm faces convex investment costs. This paper explains that: "The firm decides to invest significantly later in slightly more capacity in the convex case. This is an expected result since the investment cost the firm is facing for the convex case is significantly higher for larger investments, and therefore installing a large amount of capacity is more expensive." 


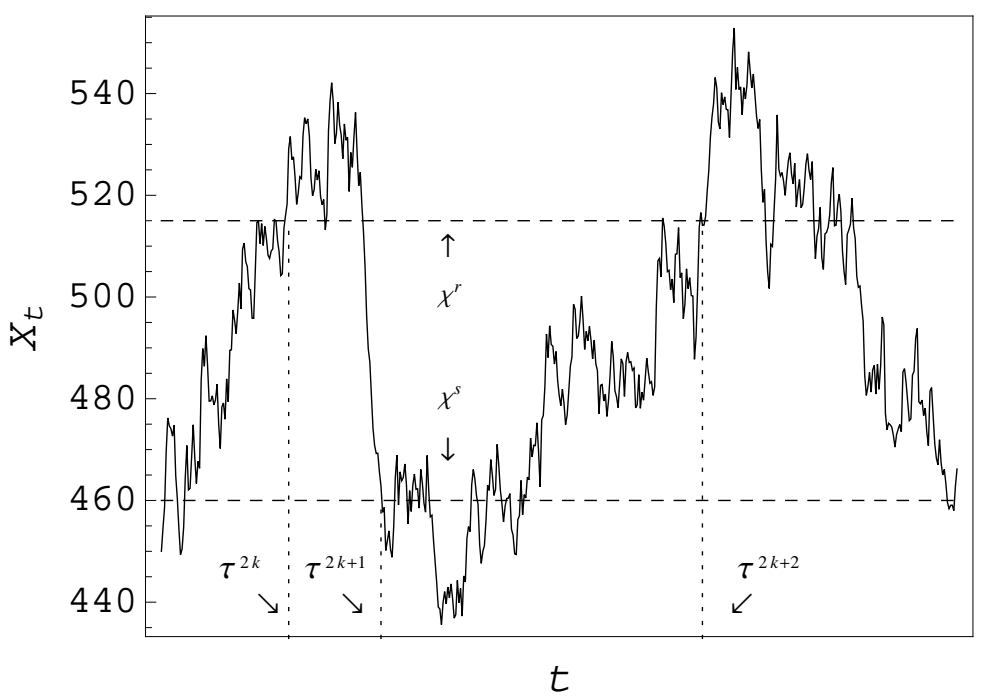

Figure 2: A simulated sample path of the uncertainty parameter, $X_{t}$, with operational triggers $\chi^{s}=460$ and $\chi^{r}=515$.

triggers, i.e., at time $t=\tau^{2 k+1}$ and $t=\tau^{2 k+2}$, respectively, for $k \in \mathbb{N}$, where $\tau^{1}>\tau^{I}$. Since there exists stationary behavior in the shut down and re-starting decisions, we assume that there is one mutual trigger for resuming operations, i.e., $X_{\tau^{2 k}}=\chi^{r}$ for all $k \in \mathbb{N}$, and one mutual trigger for suspending operations, i.e., $X_{\tau^{2 k+1}}=\chi^{s}$, for all $k \in \mathbb{N}$.

In order to find the value functions and optimal decisions of the firm, we will work backward through the timeline. Since the firm has infinitely many suspension and resumption decisions, it is not possible to consider the "last" decision of a firm. Instead, we shall consider an arbitrary point on the timeline and work backward from that moment on.

To start with, assume that production has just been suspended when the stochastic shock, $X_{t}$, dropped to the threshold $\chi^{s}$ at time $\tau^{2 k+1}$, and the firm's expected net present value (NPV) is $F^{s}\left(X_{t} ; \chi^{s}, \chi^{r}, Q\right)$, where $\chi^{s}, \chi^{r}, Q$ after the semi-colon refers to the endogenously determined optimal capacity and operational policy. Taking one step back in the timeline, we look at the decision to suspend operations. Denoting the expected NPV of a firm that is in operation by $F^{r}\left(X_{t} ; \chi^{s}, \chi^{r}, Q\right)$, we find the following:

$$
\begin{aligned}
F^{r}\left(X_{t} ; \chi^{s}, \chi^{r}, Q\right)=\frac{X_{t} Q}{\rho-\mu}- & \frac{\left(\eta Q^{2}+c Q\right)}{\rho}+ \\
& \left(\frac{X_{t}}{\chi^{s}}\right)^{\beta_{2}}\left(\frac{\left(\eta Q^{2}+c Q\right)}{\rho}-\frac{\chi^{s} Q}{\rho-\mu}-S+F^{s}\left(\chi^{s} ; \chi^{s}, \chi^{r}, Q\right)\right) .
\end{aligned}
$$

The first two terms in expression (4) reflect the expected present value of operating profits of a firm that produces forever. Since the stochastic shock to the price is growing at rate $\mu$, its discount rate differs from the rate, $\rho$, at which operating costs are discounted. At 
some point in the future when $X_{t}$ drops to $\chi^{s}$, i.e., the stochastic shock hits the suspension threshold from above signifying a sufficient reduction in the price, the firm decides to suspend operations, thereby effectively canceling out its cash flows from operating, i.e., recovering the operating costs and forgoing the revenues, but receiving the expected NPV of a suspended firm, $F^{s}\left(\chi^{s} ; \chi^{s}, \chi^{r}, Q\right)$, in exchange for switching cost $S$. The term $\left(\frac{X_{t}}{\chi^{s}}\right)^{\beta_{2}}$ can be interpreted as the expectation of a stochastic discount factor that reflects the time value of money between the current time, $t$, at which the stochastic parameter is $X_{t}$ and the future time of suspension when the stochastic parameter will hit the critical threshold $\chi^{s}$. Dixit and Pindyck (1994) (on pp. 315-316) show that $\mathbb{E}\left[e^{-\rho\left(\tau^{2 k+1}-t\right)}\right]=\left(\frac{X_{t}}{\chi^{s}}\right)^{\beta_{2}}$, where $\beta_{1}\left(\beta_{2}\right)$ is the positive (negative) root of the quadratic polynomial

$$
\frac{1}{2} \sigma^{2} \beta^{2}+\left(\mu-\frac{1}{2} \sigma^{2}\right) \beta-\rho=0 .
$$

By taking one extra step back in the decision-making timeline, we look at the decision to resume operations occurring at time $t=\tau^{2 k}$. The expected NPV of a firm that has just stopped production and now has the option to resume is given by:

$$
F^{s}\left(X_{t} ; \chi^{s}, \chi^{r}, Q\right)=\left(\frac{X_{t}}{\chi^{r}}\right)^{\beta_{1}}\left(F^{r}\left(\chi^{r} ; \chi^{s}, \chi^{r}, Q\right)-R\right)
$$

Expression (6) states that the firm obtains no profit in the suspended state, but it has an option to resume operations for a switching cost $R$ in the future.

After substitution of $F^{s}\left(\chi^{s} ; \chi^{s}, \chi^{r}, Q\right)$ from (6) in (4) and evaluating $F^{r}\left(X_{t} ; \chi^{s}, \chi^{r}, Q\right)$ for $X_{t}=\chi^{r}$ (as described in Chapter 17 of McDonald (2002)), we find an expression for $F^{r}\left(\chi^{r} ; \chi^{s}, \chi^{r}, Q\right)$ :

$$
F^{r}\left(\chi^{r} ; \chi^{s}, \chi^{r}, Q\right)=\frac{\frac{\chi^{r} Q}{\rho-\mu}-\frac{\left(\eta Q^{2}+c Q\right)}{\rho}+\left(\frac{\chi^{r}}{\chi^{s}}\right)^{\beta_{2}}\left(\frac{\chi^{s}}{\chi^{r}}\right)^{\beta_{1}}(-R)+\left(\frac{\chi^{r}}{\chi^{s}}\right)^{\beta_{2}}\left(\frac{\left(\eta Q^{2}+c Q\right)}{\rho}-\frac{\chi^{s} Q}{\rho-\mu}-S\right)}{1-\left(\frac{\chi^{r}}{\chi^{s}}\right)^{\beta_{2}}\left(\frac{\chi^{s}}{\chi^{r}}\right)^{\beta_{1}}} .
$$

Subsequently, we substitute equation (7) into (6) and evaluate $F^{s}\left(X_{t} ; \chi^{s}, \chi^{r}, Q\right)$ at $X_{t}=\chi^{s}$ :

$$
\begin{aligned}
F^{s}\left(\chi^{s} ; \chi^{s}, \chi^{r}, Q\right) & =\left(\frac{\chi^{s}}{\chi^{r}}\right)^{\beta_{1}} \\
& \left(\frac{\frac{\chi^{s} Q}{\rho-\mu}-\frac{\left(\eta Q^{2}+c Q\right)}{\rho}+\left(\frac{\chi^{r}}{\chi^{s}}\right)^{\beta_{2}}\left(\frac{\chi^{s}}{\chi^{r}}\right)^{\beta_{1}}(-R)+\left(\frac{\chi^{r}}{\chi^{s}}\right)^{\beta_{2}}\left(\frac{\left(\eta Q^{2}+c Q\right)}{\rho}-\frac{\chi^{s} Q}{\rho-\mu}-S\right)}{1-\left(\frac{\chi^{r}}{\chi^{s}}\right)^{\beta_{2}}\left(\frac{\chi^{s}}{\chi^{r}}\right)^{\beta_{1}}}\right) .
\end{aligned}
$$


We take the first-order necessary condition of $F^{r}\left(\chi^{r} ; \chi^{s}, \chi^{r}, Q\right)$ with respect to $\chi^{s}$, and the first-order necessary condition of $F^{s}\left(\chi^{s} ; \chi^{s}, \chi^{r}, Q\right)$ with respect to $\chi^{r}$. We simultaneously solve these two equations numerically to find the two switching triggers, $\chi^{s}(Q)$ and $\chi^{r}(Q)$.

The switching triggers are relevant only after the firm has made the investment decision. Now, assume a firm that has not yet invested. Here, $X_{t}$ is the level of the stochastic shock at the first moment where the firm considers investing in the market. The expected NPV of a firm that has the option to invest at $X_{t}=\chi^{I}$ in capacity $Q$ is the discounted expected value of operating forever plus the option to suspend:

$$
\begin{array}{r}
F^{I}\left(X_{t} ; \chi^{I}, \chi^{s}, \chi^{r}, Q\right)=\left(\frac{X_{t}}{\chi^{I}}\right)^{\beta_{1}}\left(\frac{\chi^{I} Q}{\rho-\mu}-\frac{\left(\eta Q^{2}+c Q\right)}{\rho}-\delta Q^{\lambda}+\left(\frac{\chi^{I}}{\chi^{s}(Q)}\right)^{\beta_{2}}\left(\frac{\left(\eta Q^{2}+c Q\right)}{\rho}\right.\right. \\
\left.\left.-\frac{\chi^{s}(Q) Q}{\rho-\mu}-S+F^{s}\left(\chi^{s}(Q) ; \chi^{s}(Q), \chi^{r}(Q), Q\right)\right)\right) .
\end{array}
$$

We find the optimal moment to invest and the corresponding optimal level of capacity by optimizing $F^{I}\left(X_{t} ; \chi^{I}, Q\right)$ with respect to $\chi^{I}$ and $Q$, thereby yielding $\chi^{I *}$ and $Q^{*}$. Due to the complexity of the first-order conditions, there is no analytical solution available for maximizing (9).

\subsection{Results}

The left panel of Figure 3 illustrates the effect of market uncertainty on the switching triggers $\chi^{s}(Q)$ and $\chi^{r}(Q)$ for a fixed level of capacity. Capacity choice $Q$ is fixed at the optimal level for $\sigma=0.10$. The result is in line with the standard real options result, viz., a higher level of uncertainty delays the firm's switching decisions. For a more uncertain market environment, the firm waits for a higher (lower) level of $X_{t}$ before it switches from the suspended (operational) state to the operational (suspended) state. The right panel of Figure 3 illustrates the effect of capacity choice $Q$ on the optimal switching triggers. Due to positive switching costs, the triggers to suspend and resume operations, respectively, lie below and above the level of $X_{t}$ for which the profit is exactly zero (indicated by the dotted line in the middle). The inverse demand function, as defined by expression (1), shows that a higher capacity level implies a higher level of $X_{t}$ is required in order to avoid a negative price. Therefore, the switching triggers increase for a higher capacity level as illustrated in Figure 3 .

Table 1 shows the combined effect of optimal capacity and timing of investment for several levels of uncertainty. Closely related to our work is the inflexible model of Hagspiel et al. (2016), in which a firm decides about the optimal investment timing and determines the 

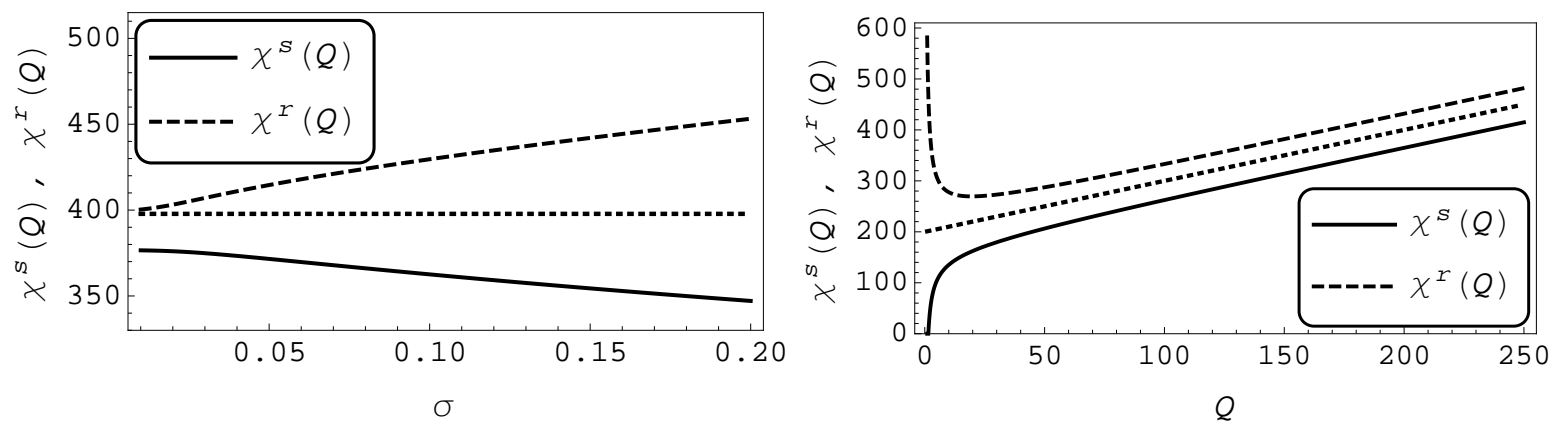

Figure 3: The effect of uncertainty $\sigma$ (left panel) and capacity choice $Q$ (right panel) on the optimal operational triggers with exogenous capacity sizes. We use the parameter combination $Q=197.81$ (left panel), $\sigma=0.1$ (right panel), $\rho=0.10, \mu=0.02, \eta=1, R=S=3000, \delta=1000, \lambda=0.7$, and $c=200$.

optimal capacity size 8 In this model, a suspension option prevents a firm from producing as soon as demand is such that price will fall below unit production costs, resulting in a costless switch of operational states. In order to compare our model with Hagspiel et al. (2016), we have chosen the same parameter values. The optimal investment strategy for our model, shown in Table 1, confirms the optimal investment strategy illustrated in Figure 6 in Hagspiel et al. (2016).

\begin{tabular}{|c||c|c|c|c|}
\hline$\sigma$ & $\chi^{I *}$ & $Q^{*}$ & $\chi^{s}\left(Q^{*}\right)$ & $\chi^{r}\left(Q^{*}\right)$ \\
\hline \hline 0.10 & 464.74 & 197.81 & 362.57 & 429.62 \\
\hline 0.15 & 678.30 & 383.19 & 547.45 & 616.33 \\
\hline 0.20 & 1321.21 & 982.31 & 1141.31 & 1221.52 \\
\hline
\end{tabular}

Table 1: Investment strategy and optimal operational triggers. Parameter values are $\rho=0.10, \mu=0.02$, $\sigma=0.10, \eta=1, c=200, S=R=3000, \delta=1000$, and $\lambda=0.7$.

The last two columns of Table 1 show the effect of increasing uncertainty on the switching triggers. In fact, these results can be explained by combining the two graphs in Figure 3 with the first two columns of Table 1. A higher level of uncertainty results in a larger capacity investment, which in turn increases both switching triggers. In effect, a firm speeds up the optimal moment to suspend production and delays the optimal moment to resume production (i.e., an indirect effect caused by the impact of uncertainty on $Q^{*}$ ). Also, we have shown in Figure 3 that a higher level of uncertainty delays both the optimal moment to suspend as well as the optimal moment to resume operations (i.e., a direct effect caused by an increase in the opportunity cost of switching). Indeed, for a fixed capacity size and positive switching

\footnotetext{
${ }^{8}$ This model is inflexible compared to another model that Hagspiel et al. (2016) define, i.e., the flexible model, in which a firm can also optimize production output after the capacity level has been determined. Specifically, with flexible production, once the capacity size has been fixed to $Q^{*}$, output can vary continuously between 0 and $Q^{*}$ depending on market conditions. By contrast, inflexible production means that output can be either 0 or $Q^{*}$, i.e., only two possible discrete values, which is also our approach.
} 
costs, our model without time lags is equivalent to the mothballing model from Chapter 7 of Dixit and Pindyck (1994). As such, we obtain similar insights about the impact of greater uncertainty on hysteresis: higher volatility pushes the operational triggers further apart, thereby delaying action. Table 1 shows that the indirect effect, stemming from the impact of uncertainty on the capacity choice, dominates the direct effect, which emanates only from uncertainty.

The impact of the switching costs on the operational triggers is illustrated in Figure 4. The first two panels allow us to compare our results with those from Chapter 7 of Dixit and Pindyck (1994). Indeed, as expected, for a given capacity, higher switching costs lead to more hysteresis. Our model is equivalent to the inflexible model of Hagspiel et al. (2016) when switching costs are zero as illustrated in the third panel of Figure 4 with $R=S=0$. Here, the firm suspends and resumes operations when the stochastic parameter becomes $\chi^{s}(Q)=\chi^{r}(Q)=397.8$, i.e., corresponding to a price that is equal to the unit production costs, i.e., $P_{t}-c=0 \Leftrightarrow X_{t}=\eta Q+c$. For positive switching costs, we find that $\chi^{s}(Q) \leq$ $397.8 \leq \chi^{r}(Q)$, i.e., the firm does not immediately suspend (resume) operations when the profit turns negative (positive) $9^{9}$ It would rather make a small operating loss than pay the switching costs (Tsekrekos, 2010). In Figure 4, we choose the optimal level of capacity, $Q$, for $R=S=3000$ and $\sigma=0.10$.

\section{Time Lags and Capacity Optimization with Operational Flexibility}

In the previous section, the firm was given the opportunity to suspend production for low prices. This section takes into account that the startup of production involves a time lag between the decision to resume and the start of the operations during which the firm is unable to obtain revenue from production. The suspension decision, on the other hand, can occur instantaneously.

\subsection{Description of the Model}

Figure 5 illustrates the decision-making timeline with time lags. We start by looking at the scenario in which the firm has just made the decision to resume operations at $t=\tau^{2 k}$. There is a necessary preparation time before a firm can obtain the "old" profit flow. Denote the deterministic time that it takes to prepare for the re-start of production, also known as the time lag, by $T \geq 0$, i.e., the firm will be operating by time $t=\tau^{2 k}+T$. If the last decision that the firm made is resumption, then the firm now has the option to suspend operations at its optimal time. It could be optimal to suspend production immediately after

\footnotetext{
${ }^{9}$ A "bang-bang" switching policy under fixed capacity occurs only with zero switching costs.
} 

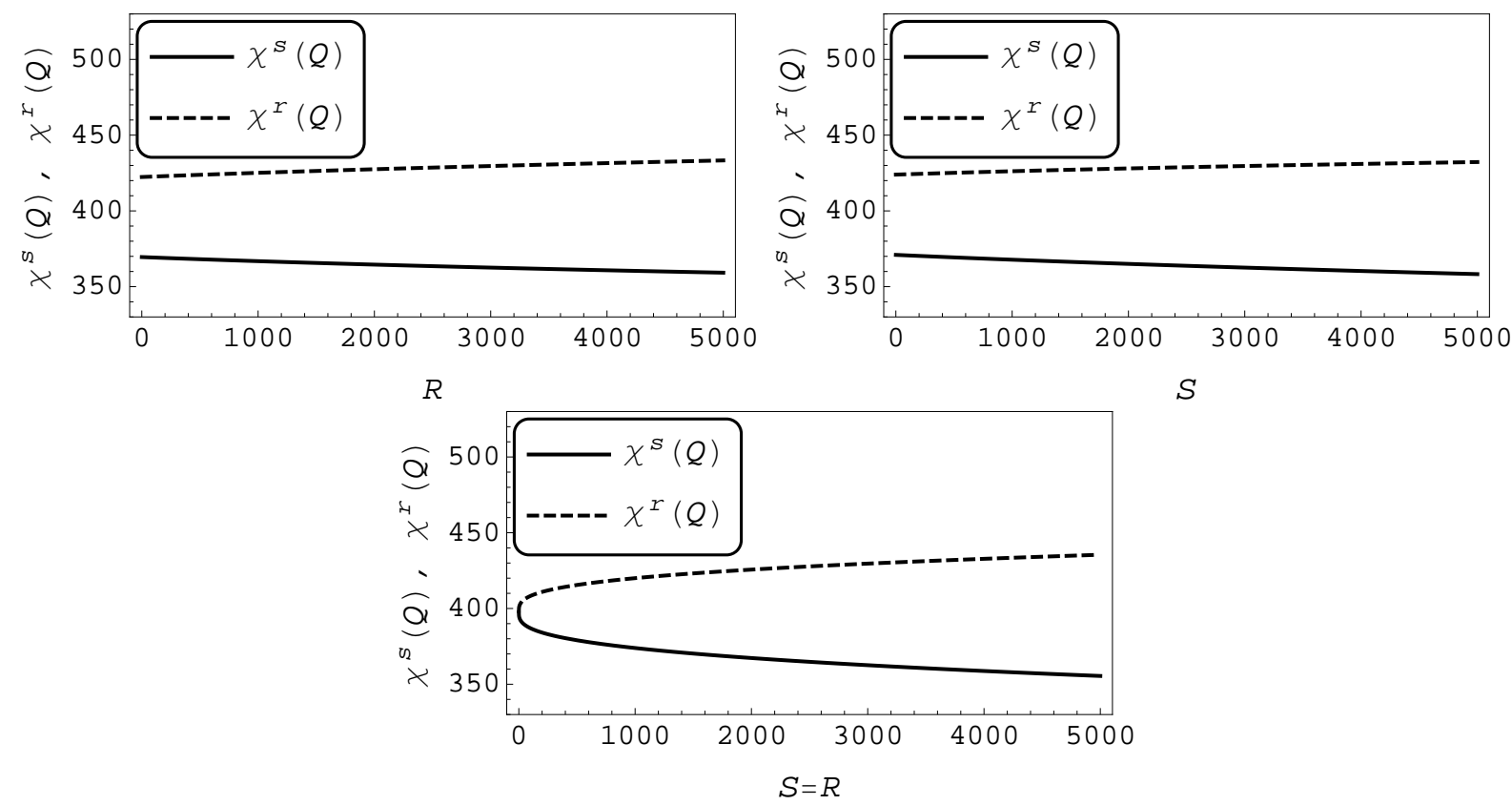

Figure 4: The effect of the switching cost parameters on the optimal operational triggers. We use the parameter combination $Q=197.81, \rho=0.10, \mu=0.02, \sigma=0.10, \eta=1, \delta=1000, \lambda=0.7$, and $c=200$. In the upper-left panel we have $S=3000$, and in the upper-right panel, we have $R=3000$.

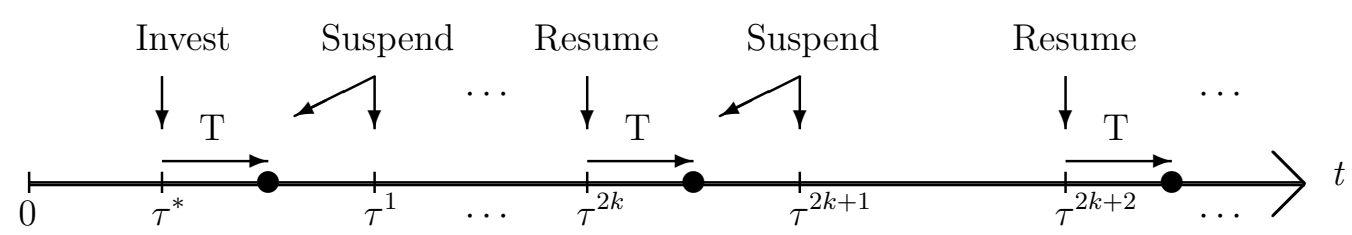

Figure 5: Decision-making timeline for investing, suspending, and resuming operations with time lags.

the time lag. In this case, the firm observes a price too low to continue production (i.e., $\left.X_{\tau^{2 k}+T} \leq X_{\tau^{2 k+1}}\right)$. Note that this implies that the firm has not yet produced anything, and it immediately goes back to the suspended state. Alternatively, the current price after the time lag is high enough to resume production (i.e., $X_{\tau^{2 k}+T}>X_{\tau^{2 k+1}}$ ), and a firm optimally suspends production later at time $t=\tau^{2 k+1}$. After one such cycle has been completed, the firm will face similar decisions in the subsequent cycle. Intuitively, a firm that has just started operations from a suspended state (i) faces a memoryless price process and (ii) has infinite shutdown/resumption options remaining. Thus, its situation is identical to that at any other point in time that it resumes operations. Likewise, a firm that has just suspended operations is in the same situation from a decision-analytic perspective at another point in time at which suspension has just occurred. Similar arguments in the literature, e.g., Chapter 7 of Dixit and Pindyck (1994) and Chapter 17 of McDonald (2002), follow from the Markovian property of the GBM and the presence of infinite subsequent operational options after each suspension or resumption decision. Due to the stationarity in these decisions, we argue that 
there is one mutual trigger for resuming operations and one mutual trigger for suspending operations, i.e., $X_{\tau^{2 k}}=\chi^{r}$ and $X_{\tau^{2 k+1}}=\chi^{s}$ for all $k \in \mathbb{N}$.

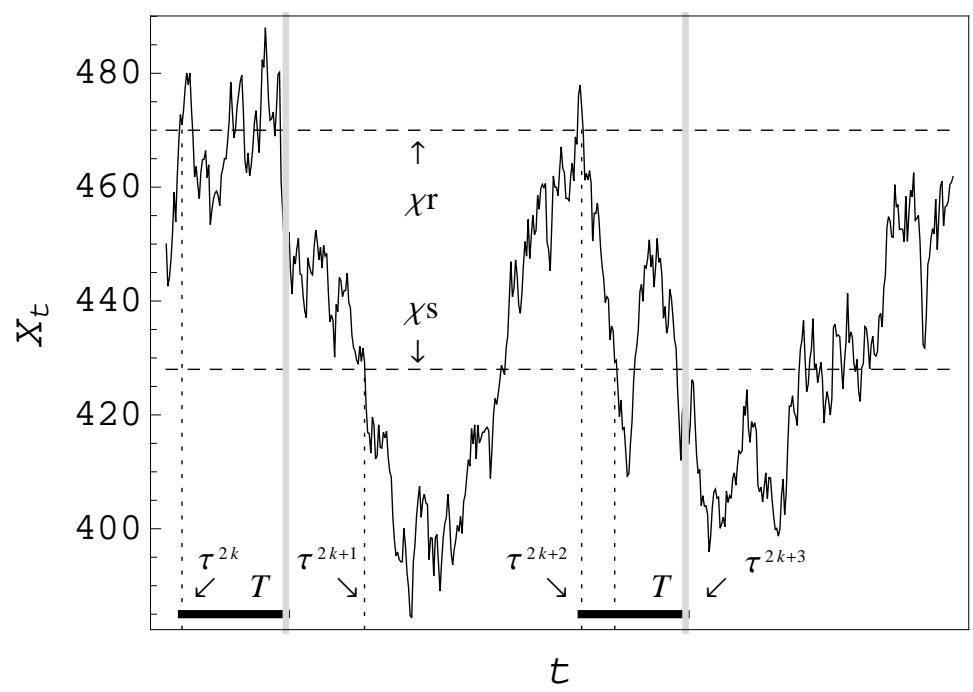

Figure 6: A simulated sample path of the uncertainty parameter, $X_{t}$. Assume operational triggers $\chi^{r}=470$ and $\chi^{s}=428$.

Figure 6 illustrates the two possible scenarios for suspending operations. Assume that the firm is initially inactive when starting to evaluate the price process. Almost immediately the firm makes the decision to resume the production at time $t=\tau^{2 k}$. After the time lag, the firm produces for a while until it is optimal to suspend the production at time $t=\tau^{2 k+1}$. At time $t=\tau^{2 k+2}$, the firm resumes the operations again, but on this occasion the price after the time lag, i.e., at $\tau^{2 k+2}+T$, is too low to produce, and production is suspended immediately.

\subsection{Suspending Operations}

In order to find the value functions and optimal decisions of the firm, we work backward through the timeline. Denote the expected NPV of a firm that has just resumed operations at time $t=\tau^{2 k+2}$ by $F^{r}\left(\chi^{r} ; \chi^{s}, \chi^{r}, Q\right)$ for some given $k \in \mathbb{N}$. By taking one step back in the decision-making timeline, we next look at the suspension decision of the firm. There are two opportunities for a firm to shut down the operation. A firm could immediately suspend after the time lag, i.e., at $t=\tau^{2 k}+T$; in this case, it holds that $X_{\tau^{2 k}+T} \leq \chi^{s}$. ${ }^{10}$ Alternatively, the firm optimally suspends operations at time $t=\tau^{2 k+1}$. To simplify the notation, from now on we shall denote $X_{\tau^{2 k}+T}$ by $X_{T}$.

\footnotetext{
${ }^{10}$ This case is peculiar since the value of $X_{t}$ at time $\tau^{2 k}+T$, i.e., right when the firm can resume production, slips below the suspension threshold, $\chi^{s}$. Figure 6 does not describe this case at time $\tau^{2 k}+T$, but it does so at time $\tau^{2 k+2}+T$.
} 
The expected NPV of an active firm at $t=\tau^{2 k}+T$ that has the opportunity to suspend is denoted by $V\left(X_{T} ; \chi^{s}, \chi^{r}, Q\right)$ and is expressed as follows:

$$
V\left(X_{T} ; \chi^{s}, \chi^{r}, Q\right)=\left\{\begin{array}{rr}
\left(\frac{X_{T}}{\chi^{r}}\right)^{\beta_{1}} F^{r}\left(\chi^{r} ; \chi^{s}, \chi^{r}, Q\right)-S-R & \text { if } X_{T} \leq \chi^{s}, \\
\frac{X_{T} Q}{\rho-\mu}-\frac{\left(\eta Q^{2}+c Q\right)}{\rho}-R+\left(\frac{X_{T}}{\chi^{s}}\right)^{\beta_{2}}\left(\frac{\left(\eta Q^{2}+c Q\right)}{\rho}-\frac{\chi^{s} Q}{\rho-\mu}\right. & \\
\left.-S+\left(\frac{\chi^{s}}{\chi^{r}}\right)^{\beta_{1}} F^{r}\left(\chi^{r} ; \chi^{s}, \chi^{r}, Q\right)\right) & \text { if } X_{T}>\chi^{s} .
\end{array}\right.
$$

In case the suspension decision is immediately performed at time $t=\tau^{2 k}+T$ (upper line of expression (10)), the firm pays switching cost $S$ for switching to the suspended state and $R$ for the previous decision to re-start the operation. Similar to Bar-Ilan and Strange (1996) and Sødal (2006), we assume that the entry costs are paid at the end of the time lag. In case the firm continues production after the time lag (lower line of expression (10)), it obtains discounted expected profit of $\frac{X_{T} Q}{\rho-\mu}-\frac{\left(\eta Q^{2}+c Q\right)}{\rho}$ plus the discounted cash flow from suspending production at time $t=\tau^{2 k+1}$ by hitting threshold $\chi^{s}$. To simplify the notation, we shall from now on denote $V\left(X_{T} ; \chi^{s}, \chi^{r}, Q\right)$ by $V\left(X_{T}\right)$.

\subsection{Resuming Operations}

Since we are working backward through the decision-making timeline, next we look at the firm's decision to resume operations at time $t=\tau^{2 k}$, which is followed by a time lag $T$. As discussed earlier, time $t=\tau^{2 k}+T$ is the first moment at which the firm can decide to suspend the production. The expected NPV of a firm that is able to resume operations is in fact just the discounted expected NPV of the active firm as in (10). This expectation is discounted back to the moment at which the operating decision is made (at $\left.t=\tau^{2 k}\right) . F^{r}\left(\chi^{r} ; \chi^{s}, \chi^{r}, Q\right)$ denotes the expected NPV of a firm that has just resumed operations at time $t=\tau^{2 k}$ and is given by the discounted expected NPV of an active firm $T$ time units later:

$$
F^{r}\left(\chi^{r} ; \chi^{s}, \chi^{r}, Q\right)=e^{-\rho T} \mathbb{E}\left[V\left(X_{T}\right) \mid \chi^{r}\right]
$$

The expected NPV of being active at time $t=\tau^{2 k}+T$ given that the decision to resume operations occurred at $t=\tau^{2 k}$ in expression (11) can be written as:

$$
\begin{aligned}
\mathbb{E}\left[V\left(X_{T}\right) \mid \chi^{r}\right]= & \int_{0}^{\chi^{s}}( \\
+\int_{\chi^{s}}^{\infty} & \left.\left(\frac{X_{T}}{\chi^{r}}\right)^{\beta_{1}} F^{r}\left(\chi^{r} ; \chi^{s}, \chi^{r}, Q\right)-R-S\right) f\left(X_{T} \mid \chi^{r}\right) d X_{T} \\
\rho & \left.\left.-\frac{\chi^{s} Q}{\rho-\mu}-S+\left(\frac{\chi^{s}}{\chi^{r}}\right)^{\beta_{1}} F^{r}\left(\chi^{r} ; \chi^{s}, \chi^{r}, Q\right)\right)\right) f\left(X_{T} \mid \chi^{r}\right) d X_{T},
\end{aligned}
$$


where $f\left(X_{T} \mid \chi^{r}\right)$ denotes the conditional probability density function at time $t=\tau^{2 k}$ of the output price at time $t=\tau^{2 k}+T$ when the production preparation is completed. According to (12), the expected NPV of an active firm $T$ time units after the decision to resume depends on the value of the stochastic process at that time: if it is less than the optimal suspension threshold, then the firm suspends operations at that time, which has an expected value captured by the first integral of 12 . Otherwise, if the value of the stochastic process $T$ time units later is greater than the optimal suspension threshold, then the firm remains operational. The expected value of such a firm is calculated by the second integral in (12). Its first three terms correspond to the discounted cash flows of a firm that operates forever, whereas the remaining terms are the discounted expected value of suspending operations in the future if the value of the stochastic process were to drop to the suspension threshold followed by subsequent resumption. Equation (12) can be re-written as:

$$
\begin{aligned}
\mathbb{E}\left[V\left(X_{T}\right) \mid \chi^{r}\right]= & \left(\frac{\chi^{r}}{\chi^{r}}\right)^{\beta_{1}} F^{r}\left(\chi^{r} ; \chi^{s}, \chi^{r}, Q\right) \Phi\left(v\left(\chi^{s}, \chi^{r}\right)-\beta_{1} \sigma \sqrt{T}\right) e^{\rho T} \\
- & (S+R) \Phi\left(v\left(\chi^{s}, \chi^{r}\right)\right)+\frac{\chi^{r} Q}{\rho-\mu}\left(1-\Phi\left(v\left(\chi^{s}, \chi^{r}\right)-\sigma \sqrt{T}\right)\right) e^{\mu T} \\
- & \left(\frac{\left(\eta Q^{2}+c Q\right)}{\rho}+R\right)\left(1-\Phi\left(v\left(\chi^{s}, \chi^{r}\right)\right)\right)+\left(\frac{\chi^{r}}{\chi^{s}}\right)^{\beta_{2}}\left(\frac{\left(\eta Q^{2}+c Q\right)}{\rho}\right. \\
& \left.-\frac{\chi^{s} Q}{\rho-\mu}-S+\left(\frac{\chi^{s}}{\chi^{r}}\right)^{\beta_{1}} F^{r}\left(\chi^{r} ; \chi^{s}, \chi^{r}, Q\right)\right)\left(1-\Phi\left(v\left(\chi^{s}, \chi^{r}\right)-\beta_{2} \sigma \sqrt{T}\right)\right) e^{\rho T},
\end{aligned}
$$

where $\Phi(\cdot)$ is the standard normal cumulative distribution function and $v\left(\chi^{s}, \chi^{r}\right)$ is defined by

$$
v\left(\chi^{s}, \chi^{r}\right)=\frac{\log \left(\chi^{s}\right)-\log \left(\chi^{r}\right)-\left(\mu-\frac{1}{2} \sigma^{2}\right) T}{\sigma \sqrt{T}} .
$$

Appendix B explains the derivation of expression (13) from expression (12). Intuitively, (13) calculates the integrals using the cumulative distribution of a standard normal random variable, $\Phi(\cdot)$, and the critical value, $v(\cdot)$, which are standard expressions related to the conditional probabilities for hitting a given threshold within a specified amount of time for the standard normal random variable (Etheridge, 2002). The first term of (13) indicates that the firm's value $T$ time units later would be the expected NPV of a suspended firm with probability $\Phi\left(v\left(\chi^{s}, \chi^{r}\right)-\beta_{1} \sigma \sqrt{T}\right)$, i.e., the probability that the value of the stochastic process drops below the suspension threshold in the next $T$ time units. Similarly, the next four terms in (13) correspond to the expected NPV of an active firm with certain probabilities, i.e., that the value of the stochastic process remains above the suspension threshold in the next $T$ time units:

1. $-(S+R) \Phi\left(v\left(\chi^{s}, \chi^{r}\right)\right)$ is the expected switching cost given that the value of the stochastic process drops below the suspension threshold in the next $T$ time units. 
2. $\frac{\chi^{r} Q}{\rho-\mu}\left(1-\Phi\left(v\left(\chi^{s}, \chi^{r}\right)-\sigma \sqrt{T}\right)\right) e^{\mu T}$ is the expected revenue that the firm obtains from operating indefinitely provided that the value of the stochastic process does not drop below the suspension threshold in the next $T$ time units.

3. $-\left(\frac{\left(\eta Q^{2}+c Q\right)}{\rho}+R\right)\left(1-\Phi\left(v\left(\chi^{s}, \chi^{r}\right)\right)\right)$ is the expected cost of operating indefinitely provided that the value of the stochastic process does not drop below the suspension threshold in the next $T$ time units.

4. $\left(\frac{\chi^{r}}{\chi^{s}}\right)^{\beta_{2}}\left(\frac{\left(\eta Q^{2}+c Q\right)}{\rho}-\frac{\chi^{s} Q}{\rho-\mu}-S+\left(\frac{\chi^{s}}{\chi^{r}}\right)^{\beta_{1}} F^{r}\left(\chi^{r} ; \chi^{s}, \chi^{r}, Q\right)\right)\left(1-\Phi\left(v\left(\chi^{s}, \chi^{r}\right)-\beta_{2} \sigma \sqrt{T}\right)\right) e^{\rho T}$ is the expected NPV from recovered operating costs and forgone revenues plus the value to resume operations as a result of subsequent suspension given that the value of the stochastic process does not drop below the suspension threshold in the next $T$ time units.

The following proposition explains that for $T$ very close to zero, the model described in this section simplifies to the model of Section 3 (see Appendix C for the proof).

Proposition 1. The value functions of the model that incorporate time lag $T$ converge to the value functions without a time lag, as $T \rightarrow 0$.

\subsection{Switching Triggers}

Following Chapter 17 of McDonald (2002), we substitute expression (13) into (11). Solving for $F^{r}\left(\chi^{r} ; \chi^{s}, \chi^{r}, Q\right)$ yields the expression:

$$
\begin{aligned}
& F^{r}\left(\chi^{r} ; \chi^{s}, \chi^{r}, Q\right) \\
= & \frac{e^{-\rho T}}{1-\Phi\left(v\left(\chi^{s}, \chi^{r}\right)-\beta_{1} \sigma \sqrt{T}\right)-\left(1-\Phi\left(v\left(\chi^{r}\right)-\beta_{2} \sigma \sqrt{T}\right)\right)\left(\frac{\chi^{s}}{\chi^{r}}\right)^{\beta_{1}}\left(\frac{\chi^{r}}{\chi^{s}}\right)^{\beta_{2}}} \\
& \times\left[-(S+R) \Phi\left(v\left(\chi^{s}, \chi^{r}\right)\right)+\frac{\chi^{r} Q}{\rho-\mu}\left(1-\Phi\left(v\left(\chi^{s}, \chi^{r}\right)-\sigma \sqrt{T}\right)\right) e^{\mu T}\right. \\
& -\left(\frac{\left(\eta Q^{2}+c Q\right)}{\rho}+R\right)\left(1-\Phi\left(v\left(\chi^{s}, \chi^{r}\right)\right)\right)+\left(\frac{\chi^{r}}{\chi^{s}}\right)^{\beta_{2}} \\
& \left.\left(\frac{\left(\eta Q^{2}+c Q\right)}{\rho}-\frac{\chi^{s} Q}{\rho-\mu}-S\right)\left(1-\Phi\left(v\left(\chi^{s}, \chi^{r}\right)-\beta_{2} \sigma \sqrt{T}\right)\right) e^{\rho T}\right] .
\end{aligned}
$$

This closed-form solution for $F^{r}\left(\chi^{r} ; \chi^{s}, \chi^{r}, Q\right)$ is substituted into the value function of a suspended firm, i.e.,

$$
F^{s}\left(\chi^{s} ; \chi^{s}, \chi^{r}, Q\right)=\left(\frac{\chi^{s}}{\chi^{r}}\right)^{\beta_{1}} F^{r}\left(\chi^{r} ; \chi^{s}, \chi^{r}, Q\right)
$$


Next, we take the first-order condition of $F^{r}\left(\chi^{r} ; \chi^{s}, \chi^{r}, Q\right)$ with respect to $\chi^{s}$ and the firstorder condition of $F^{s}\left(\chi^{s} ; \chi^{s}, \chi^{r}, Q\right)$ with respect to $\chi^{r}$ :

$$
\begin{aligned}
& \frac{\partial F^{r}\left(\chi^{r} ; \chi^{s}, \chi^{r}, Q\right)}{\partial \chi^{s}}=0, \\
& \frac{\partial F^{s}\left(\chi^{s} ; \chi^{s}, \chi^{r}, Q\right)}{\partial \chi^{r}}=0 .
\end{aligned}
$$

These equations are solved simultaneously numerically for $\chi^{s}$ and $\chi^{r}$ for every $Q \geq 0$. Solutions are denoted by $\chi^{s}(Q)$ and $\chi^{r}(Q)$.

\subsection{Investment Trigger and Capacity Choice}

Suppose that the firm has not yet invested in the market. The option value of the firm that invests at $\chi^{I}$ in capacity $Q$ is given by

$$
\begin{aligned}
& F^{I}\left(X_{t} ; \chi^{I}, \chi^{s}, \chi^{r}, Q\right)=e^{-\rho T}\left(\frac{X_{t}}{\chi^{I}}\right)^{\beta_{1}}\left[\left(\frac{X_{T}}{\chi^{r}(Q)}\right)^{\beta_{1}} F^{r}\left(\chi^{r}(Q) ; \chi^{s}(Q), \chi^{r}(Q), Q\right)\right. \\
\times & \Phi\left(v\left(\chi^{s}(Q), X_{T}\right)-\beta_{1} \sigma \sqrt{T}\right) e^{\rho T}-(S) \Phi\left(v\left(\chi^{s}(Q), X_{T}\right)\right) \\
+ & \frac{X_{T} Q}{\rho-\mu}\left(1-\Phi\left(v\left(\chi^{s}(Q), X_{T}\right)-\sigma \sqrt{T}\right)\right) e^{\mu T}-\left(\frac{\left(\eta Q^{2}+c Q\right)}{\rho}\right)\left(1-\Phi\left(v\left(\chi^{s}(Q), X_{T}\right)\right)\right) \\
+ & \left(\frac{X_{T}}{\chi^{s}(Q)}\right)^{\beta_{2}}\left(\frac{\left(\eta Q^{2}+c Q\right)}{\rho}-\frac{\chi^{s}(Q) Q}{\rho-\mu}-S+\left(\frac{\chi^{s}(Q)}{\chi^{r}(Q)}\right)^{\beta_{1}} F^{r}\left(\chi^{r}(Q) ; \chi^{s}(Q), \chi^{r}(Q), Q\right)\right) \\
\times & \left.\left(1-\Phi\left(v\left(\chi^{s}(Q), X_{T}\right)-\beta_{2} \sigma \sqrt{T}\right)\right) e^{\rho T}-\delta Q^{\lambda}\right] .
\end{aligned}
$$

The $\left(\frac{X_{t}}{\chi^{I}}\right)^{\beta_{1}}$ and $e^{-\rho T}$ terms are (expected) discount factors reflecting the time value of money from the current point in time to investment and from investment to the start of cash flows, respectively. Once investment has been triggered and the time to build elapsed, the firm can either proceed to earn cash flows or suspend operations. The first term in the square brackets corresponds to the latter situation, i.e., the value of the stochastic shock $T$ time units after the investment decision has been taken is too low to warrant production. Thus, as in (13), the firm suspends operations. However, if the stochastic shock $T$ time units after investment remains sufficiently high, then the firm proceeds with production until the stochastic shock becomes low enough to suspend operations. The terms in the third to fifth lines encompass this situation, which again mirrors that in (13). Finally, the investment cost is subtracted from the cash flows as the last term in (19). As in Section 3 , the optimal capacity and investment trigger are found by taking the first-order condition of expression 
(19) with respect to $\chi^{I}$ and $Q$. The optimal values are denoted by $\chi^{I *}$ and $Q^{*}$. Substitution of $Q^{*}$ in $\chi^{s}(Q)$ and $\chi^{r}(Q)$ yields $\chi^{s}\left(Q^{*}\right)$ and $\chi^{r}\left(Q^{*}\right)$, respectively.

\section{Numerical Examples}

In order to compare the results between models with (Section 4) and without (Section 3) time lags, we consider the set of parameters from Section 3 . Note that our model simplifies to the model of Bar-Ilan and Strange (1996) and Sødal (2006) by choosing $\eta=0$ and $Q=1$. Even though we take a slightly different version of the discount factor approach of Sødal (2006), we can replicate his results (Table 1, p. 1972) by making the same parameter assumptions, which can be seen as a validation exercise for our model.

\subsection{Value Functions}

The value functions $F^{s}\left(X_{t} ; \chi^{s}, \chi^{r}, Q\right)-\delta Q^{\lambda}, F^{r}\left(X_{t} ; \chi^{s}, \chi^{r}, Q\right)-\delta Q^{\lambda}$, and $F^{I}\left(X_{t} ; \chi^{I}, Q\right)$ are shown in Figure 7. Note that investment costs are subtracted from operational and suspended value functions in order to value match with the option value to invest, $F^{I}\left(X_{t} ; X_{T}, Q\right)$. Due to the assumption that the entry cost is paid after the time lag, there is a gap in the value of $R+S=6000$ at $X_{t}=\chi^{s}$. However, when the stochastic shock, $X_{t}$, hits $\chi^{r}$, the operating and suspended value functions value match perfectly. The numerical example from Figure 7 assumes that there is no time lag 11

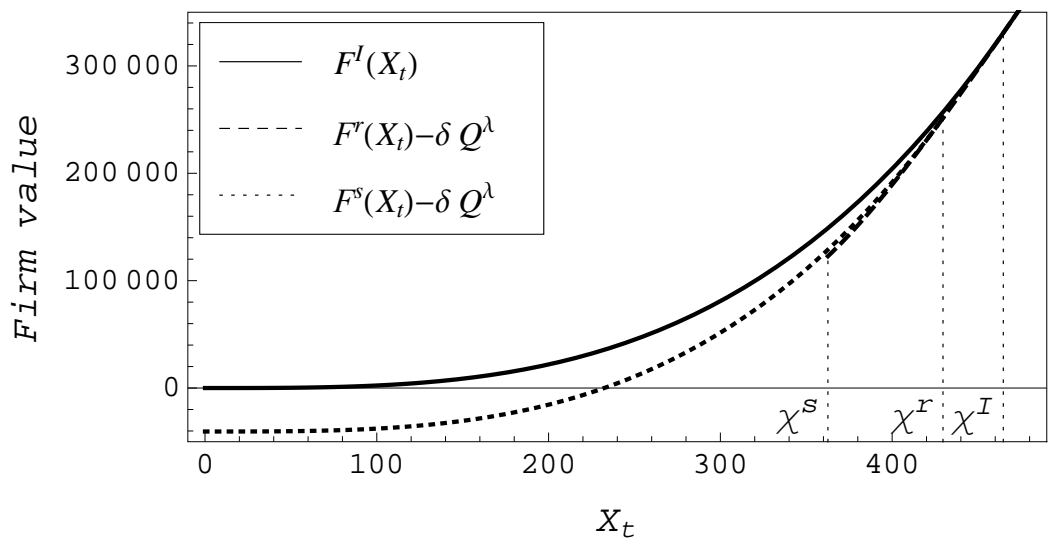

Figure 7: Value of the investment opportunity. Capacity $Q^{*}=197.81$ and triggers $\chi^{s}=362.6, \chi^{r}=429.6$, and $\chi^{I}=464.7$ are optimal under parameter combination $\rho=0.10, \mu=0.02, \sigma=0.10, \eta=1, R=S=3000$, $\delta=1000, \lambda=0.7, c=200$, and $T=0$.

\footnotetext{
${ }^{11}$ When $\delta=82.16$, the investment and resumption thresholds both equal 402.499 with an investment cost of 3000.14. Subsequent decreases to $\delta$ will actually result in an investment threshold that is lower than the one for resumption. This unusual result would generally occur only if the investment cost were less than the resumption cost, which is unlikely because it requires a facility's cost of resuming operations to be on level pegging as the overnight capital cost of a new facility.
} 


\subsection{Switching Triggers}

Consider a firm that has invested and has a known total capacity. Figure 8 illustrates the effect of uncertainty (right panel) and the length of the time lag (left panel) on the optimal switching triggers. The superscript lag highlights the results that incorporate the time lag. We find that the time lag speeds up the decision to resume operations whenever a firm is in the suspended state but delays the suspension of operations from an active state.
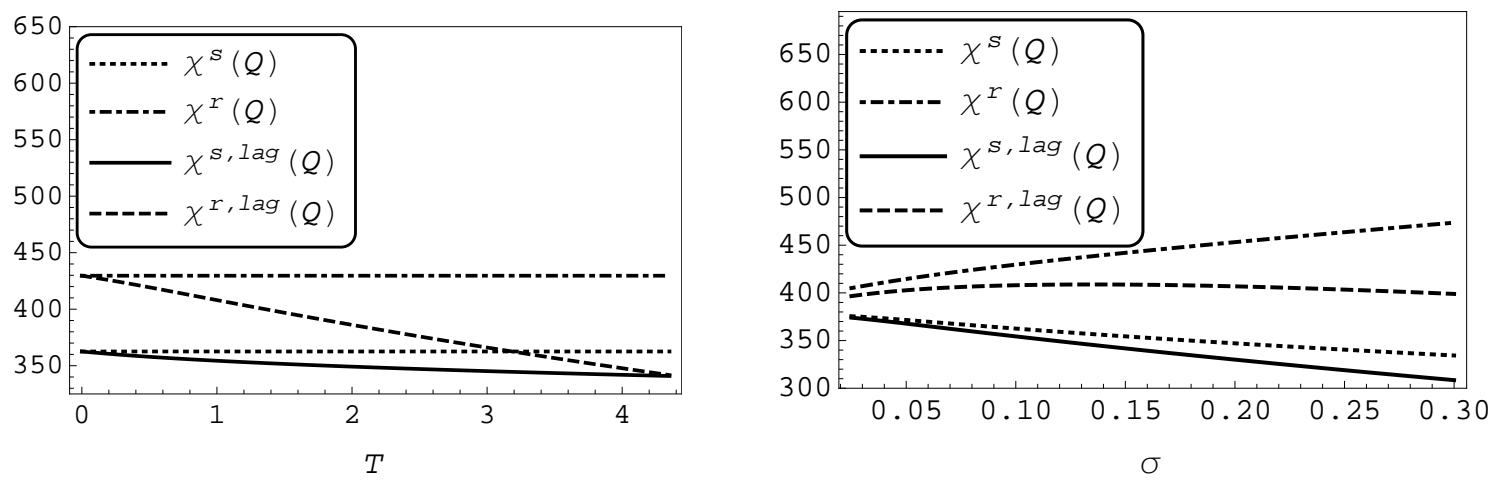

Figure 8: The effect of time lag $T$ and uncertainty $\sigma$ on the optimal triggers $\chi^{r}(Q), \chi^{s}(Q), \chi^{r, l a g}(Q)$, and $\chi^{s, l a g}(Q)$. Take parameter combination $Q=197.81, \rho=0.10, \mu=0.02, \sigma=0.10, \eta=1, R=S=3000$, $\delta=1000, \lambda=0.7, c=200$, and $T=1$. Capacity choice, $Q$, is optimal for $T=0$ and $\sigma=0.10$.

First, consider the decision to resume operations. The firm has the possibility to suspend operations for low prices; however, the upward potential of the market is unlimited. Thus, the firm is expected to have a higher profit after the time lag. At the moment that the firm decides to resume operations, it has to wait for $T$ time units before it actually gains revenue. As a result, it expects that after the time lag, the price will be already higher than would be optimal. Taking this into account, it is optimal to make the decision to resume production a little earlier. A positive market drift only strengthens this result 12 Second, the time lag slightly delays the optimal moment to suspend operations. Assume a firm that has to wait for a few months before it gains revenue from the production facility. Once it is in production, it will hesitate to suspend the production facility since it knows that this decision will be followed by another start of the operation that includes an additional time lag.

We find that the time lag has a stronger influence on the resumption decision than on the suspension one. This is because operations are forced to be inactive for at least the duration of this time lag. Besides the switching cost that has to be paid from going from one state to the other, the length of the time lag is another "burden" for the firm because it cannot

\footnotetext{
${ }^{12}$ Also, under the assumption of a negative market drift, the firm resumes operations at a lower trigger when it faces a larger time lag. However, this trigger does not diminish as much compared to the case of a positive market drift. Similarly, the trigger to suspend the production does not increase as much for a larger time lag under a negative drift compared to the case with a positive drift.
} 
receive any (potentially high) revenue in this period.13 Hence, relative to a model without time lags, e.g., Dixit and Pindyck (1994), we demonstrate that hysteresis, i.e., an increase in the delay between switching operational states in response to higher uncertainty, is actually weakened as a result of incorporating time lags.

The right panel of Figure 8 confirms the result found in Bar-Ilan and Strange (1996) and Sødal (2006), i.e., higher uncertainty might hasten a firm's entry decision. Bar-Ilan and Strange (1996) explain that there are two effects, where the level of uncertainty determines which one dominates. First, an increase in uncertainty raises the expected profit over the time lag period, which might result in an earlier entry. The expected profits can increase due to the availability of a subsequent abandonment option that truncates the downside of the market. Second, a higher level of uncertainty increases the likelihood of bad news, which delays the firm's decision to resume operations.

\subsection{Investment Decision}

\begin{tabular}{llcccc}
\hline$\sigma$ & $T$ & $\chi^{I *, \operatorname{lag}}$ & $Q^{* \operatorname{lag}}$ & $\chi^{\text {slag }}\left(Q^{* \operatorname{lag}}\right)$ & $\chi^{r, \operatorname{lag}}\left(Q^{* \operatorname{lag}}\right)$ \\
\hline 0.1 & 0 & 464.7 & 197.8 & 362.6 & 429.6 \\
0.1 & 0.1 & 470.9 & 202.5 & 366.2 & 432.4 \\
0.1 & 0.3 & 469.2 & 203.1 & 364.8 & 428.8 \\
0.1 & 0.5 & 469.9 & 204.0 & 363.9 & 425.2 \\
\hline 0.15 & 0 & 678.3 & 383.2 & 547.5 & 616.3 \\
0.15 & 0.1 & 682.6 & 388.4 & 539.8 & 627.9 \\
0.15 & 0.3 & 678.1 & 390.5 & 536.0 & 616.2 \\
0.15 & 0.5 & 673.9 & 393.3 & 534.2 & 604.7 \\
\hline 0.2 & 0 & 1321.2 & 982.3 & 1119.8 & 1247.1 \\
0.2 & 0.1 & 1317.9 & 991.9 & 1113.2 & 1225.6 \\
0.2 & 0.3 & 1295.9 & 1004.6 & 1106.9 & 1174.1 \\
0.2 & 0.5 & 1274.7 & 1017.7 & 1106.5 & 1130.4 \\
\hline
\end{tabular}

Table 2: Investment strategy and optimal operational triggers. Parameter values are $\rho=0.10, \mu=0.02, \eta=1$, $c=200, S=R=3000, \lambda=0.7$, and $\delta=1000$.

We contribute to the literature by incorporating an initial investment decision in which the firm has the additional option to optimize capacity. Table 2 shows for several levels of

\footnotetext{
${ }^{13}$ For a very large time lag, the firm is not at all motivated to suspend operations. In the case of unrealistically large time lags, e.g., $T=4.4$ years, the switching triggers do not exist, and the firm does not use the opportunity to suspend operations. The switching triggers, $\chi^{s}$ and $\chi^{r}$, in Figure 8 are relatively large compared to those in Bar-Ilan and Strange (1996) and Sødal (2006) due to the higher assumed level of unit production choice. By definition of the GBM, we know that higher levels of $X_{t}$ result in larger shocks. Therefore, we find in our example that for a time lag larger than 4.4 years, a firm will not use the opportunity to enter and exit the market, whereas for the examples in the other two mentioned papers, this result has not yet occurred after 8 years.
} 
uncertainty the effect of the time lag on the optimal investment decisions and the switching triggers. The conventional result is that a larger capacity investment corresponds to a delay in investment timing. This result still holds when an increase in uncertainty is considered. However, where a longer time lag results in an increase in the size of investment, it does not necessarily lead to a higher investment trigger. This result is further strengthened for a higher level of uncertainty. The larger capacity level is related to the expected profit of the firm over the time lag. Specifically, the ability of a firm to suspend operations means that the downside of the investment is truncated. Therefore, the firm expects revenues to increase during the time lag, thereby resulting in a larger expected profit once the firm can pursue the production after the delay. Hence, a longer time lag further strengthens this result, which justifies the larger capacity choice.

Result 1. Under capacity optimization, an increase in the length of the time lag results in a larger capacity size.

The effect of the time lag on the optimal timing of investment is ambiguous. In particular, it can be optimal to delay investment due to the larger capacity choice (Dangl, 1999). Alternatively, the firm may hasten investment because of the first time lag right after the investment decision. As explained in the left panel of Figure 8, increasing the length of the time lag makes a firm hasten the entry decision.

Capacity optimization also affects the switching triggers after entry, as is shown in the last two columns of Table 2, From Figure 8, we know that when capacity optimization is not an issue, a longer time lag reduces the level of both triggers, i.e., the direct effect of an increasing time lag. Yet, the switching triggers are also indirectly affected by the time lag, i.e., via the capacity level. Result 1 indicates that an increase in the length of the time lag results in a higher capacity level, which, in turn, increases the level of the switching triggers. Recall that a higher capacity level implies a higher level of $X_{t}$ for which production becomes (un)profitable (see the right panel of Figure 3). The indirect effect dominates only for a small initial level of the time lag and a relatively low uncertainty (Table 2).

Result 2. Under capacity optimization, a longer time lag leads to higher investment and operational triggers only when uncertainty is relatively low and the initial time lag is small.

\subsection{Counterfactual Effects}

We assess the impact of the time lag on the value of the firm by performing a counterfactual analysis. Specifically, we assume that a firm neglects the time lag when it decides about the optimal triggers for suspending and resuming operations, i.e., it uses the triggers that do not incorporate the time lag from Section 3 . The counterfactual analysis determines 
the percentage loss in value when the firm does not suspend and resume operations at the optimal triggers due to neglect of the time lag as follows:

$$
\begin{aligned}
D^{r}\left(Q^{*}, Q^{* l a g}\right)=\frac{F^{r}\left(\chi^{r, l a g}\left(Q^{* l a g}\right) ; \chi^{s, l a g}\left(Q^{* l a g}\right), \chi^{r, l a g}\left(Q^{* l a g}\right), Q^{* l a g}\right)-\delta Q^{* l a g} \lambda}{F^{r}\left(\chi^{r, l a g}\left(Q^{* l a g}\right) ; \chi^{s}\left(Q^{*}\right), \chi^{r}\left(Q^{*}\right), Q^{*}\right)-\delta Q^{* \lambda}} \\
-\frac{F^{r}\left(\chi^{r, l a g}\left(Q^{* l a g}\right) ; \chi^{s}\left(Q^{*}\right), \chi^{r}\left(Q^{*}\right), Q^{*}\right)-\delta Q^{* \lambda}}{F^{r}\left(\chi^{r, l a g}\left(Q^{* l a g}\right) ; \chi^{s}\left(Q^{*}\right), \chi^{r}\left(Q^{*}\right), Q^{*}\right)-\delta Q^{* \lambda}},
\end{aligned}
$$

and

$$
\begin{aligned}
D^{s}\left(Q^{*}, Q^{* l a g}\right)=\frac{F^{s}\left(\chi^{s, l a g}\left(Q^{* l a g}\right) ; \chi^{s, l a g}\left(Q^{* l a g}\right), \chi^{r, \operatorname{lag}}\left(Q^{* l a g}\right), Q^{* l a g}\right)-\delta Q^{* l a g} \lambda}{F^{s}\left(\chi^{s, l a g}\left(Q^{* l a g}\right) ; \chi^{s}\left(Q^{*}\right), \chi^{r}\left(Q^{*}\right), Q^{*}\right)-\delta Q^{* \lambda}} \\
-\frac{F^{s}\left(\chi^{s, l a g}\left(Q^{* l a g}\right) ; \chi^{s}\left(Q^{*}\right), \chi^{r}\left(Q^{*}\right), Q^{*}\right)-\delta Q^{* \lambda}}{F^{s}\left(\chi^{s, l a g}\left(Q^{* l a g}\right) ; \chi^{s}\left(Q^{*}\right), \chi^{r}\left(Q^{*}\right), Q^{*}\right)-\delta Q^{* \lambda}} .
\end{aligned}
$$

$D^{r}\left(Q^{*}, Q^{* l a g}\right)$ and $D^{s}\left(Q^{*}, Q^{* l a g}\right)$ reflect the counterfactual value losses of resuming and suspending operations, respectively ${ }^{14}$ Table 3 includes the counterfactual values to the results in Table 2 .

\begin{tabular}{llcc}
\hline$\sigma$ & $T$ & $D^{s}\left(Q^{*}, Q^{* \operatorname{lag}}\right)$ & $D^{r}\left(Q^{*}, Q^{\text {*lag }}\right)$ \\
\hline 0.1 & 0 & $0 \%$ & $0 \%$ \\
0.1 & 0.1 & $0.008 \%$ & $0.002 \%$ \\
0.1 & 0.3 & $0.075 \%$ & $0.023 \%$ \\
0.1 & 0.5 & $0.213 \%$ & $0.068 \%$ \\
\hline 0.15 & 0 & $0 \%$ & $0 \%$ \\
0.15 & 0.1 & $0.017 \%$ & $0.009 \%$ \\
0.15 & 0.3 & $0.152 \%$ & $0.088 \%$ \\
0.15 & 0.5 & $0.399 \%$ & $0.240 \%$ \\
\hline 0.2 & 0 & $0 \%$ & $0 \%$ \\
0.2 & 0.1 & $0.054 \%$ & $0.042 \%$ \\
0.2 & 0.3 & $0.366 \%$ & $0.298 \%$ \\
0.2 & 0.5 & $0.808 \%$ & $0.673 \%$ \\
\hline
\end{tabular}

Table 3: Uncertainty, time lags, and counterfactual effects. Parameter values are $\rho=0.10, \mu=0.02, \eta=1$, $c=200, S=R=3000, \lambda=0.7$, and $\delta=1000$.

As expected, the percentage loss in value due to the neglect of the time lag increases with the length of the time lag. When a firm resumes operations at the "wrong" (i.e., too high) trigger, it misses revenue due to the forced time lag. An increase in the market uncertainty

\footnotetext{
${ }^{14}$ Substitute $X_{t}=\chi^{r, l a g}\left(Q^{* l a g}\right)$ into $F^{r}\left(X_{t} ; \chi^{s}, \chi^{r}, Q\right)-\delta Q^{\lambda}$ for the counterfactual value loss $D^{r}\left(Q^{*}, Q^{* l a g}\right)$ because the firm optimizes the option value to resume operations at some moment in the future under the assumption that it has just stopped producing. For similar reasons, we substitute $X_{t}=\chi^{s, l a g}\left(Q^{* l a g}\right)$ into $F^{s}\left(X_{t} ; \chi^{s}, \chi^{r}, Q\right)-\delta Q^{\lambda}$ for the counterfactual value loss $D^{s}\left(Q^{*}, Q^{* l a g}\right)$.
} 
also increases the counterfactual effects. This is indirectly caused by the increase in the optimal capacity choice. For a firm with a high production capacity, it is very costly to continue production too long with a negative revenue. Similarly, a firm misses out on high profits when the decision to continue the production is made later than optimal. Especially for a very uncertain market environment and a long time lag, it is important for a firm to incorporate the time lag in the model in order to find the correct triggers. The counterfactual values may seem very low; however, under high uncertainty, the capacity level (and, thus, the production level) is such that $0.8 \%$ of the total firm value is a big gain in revenue. Low counterfactual percentage values are also observed in more realistic case studies. For example, in an analysis of U.K. transmission investment under uncertainty, van der Weijde and Hobbs (2012) find that the expected cost of ignoring uncertainty is modest in percentage terms, i.e., $0.08 \%$. Nevertheless, they argue that this loss could be a non-negligible portion of the planner's budget.

Since neither Bar-Ilan and Strange (1996) nor Sødal (2006) performs a counterfactual analysis in the model with a fixed capacity, we conduct this in Table 4 . The style of Table 4 is similar to the one that is presented in Bar-Ilan and Strange (1996) and Sødal (2006); therefore, it considers $\sigma^{2}$ rather than $\sigma$. Our model can be simplified to their model by setting $Q=1$ and $\eta=0$, i.e., by removing capacity sizing as a decision variable ${ }^{15}$ Even so, analytical solutions are not possible: indeed, the model of Bar-Ilan and Strange (1996) requires numerical solutions. In general, we would have to remove switching costs and time lags in order to solve the model analytically.

Result 3. There exists a parabolic effect in $\sigma$ in the counterfactual value losses when capacity is not optimized. For intermediate levels of market uncertainty, the firm has the most benefit from correctly choosing the triggers for which it resumes or suspends the operation.

Contrary to the model where capacity is optimized, we find that there is a parabolic effect in the counterfactual value losses. Namely, for relatively low levels of uncertainty, the firm is more confident that the price after the time lag is still high enough to pursue the production, and, for very high levels of uncertainty, the price level is so unpredictable that the exact switching triggers matter less. The price is expected to reach a large bandwidth within a short time frame anyway. However, we observe that when the firm is given the opportunity to optimize its capacity level, a higher level of uncertainty corresponds to a steep increase in the capacity level. When a large capacity level is involved, it contributes to the importance to choose the correct triggers, thereby resulting in a larger counterfactual effect. Hence,

15 Bar-Ilan and Strange (1996) consider the same set of parameters; however, Sødal (2006) detects a small technical error in this analysis of Bar-Ilan and Strange (1996) and rectifies the results. 


\begin{tabular}{|c|c|c|c|c|c|c|}
\hline$\sigma^{2}$ & \multicolumn{2}{|c|}{$T=0$} & \multicolumn{2}{|c|}{$T=6$} & & \\
\hline & $\chi^{s}$ & $\chi^{r}$ & s,lag & $X^{r}$, & $\bar{D}^{s}(\cdot)$ & $D^{r}(\cdot)$ \\
\hline 0.00 & 1.000 & 1.025 & 000 & 1.025 & & $0.000 \%$ \\
\hline .01 & & & & & & \\
\hline 02 & 0.7 & 1.3 & & & & \\
\hline 03 & 0 & 1.3 & & & & \\
\hline & & & & & & \\
\hline & & & & & & \\
\hline & & & & & & \\
\hline & 0 & 1. & & & & 1.74 \\
\hline & 587 & 1. & & & & $1.700 \%$ \\
\hline & 56 & 2.0 & & & $\%$ & $1.635 \%$ \\
\hline & 0.5 & 2.2 & & & & $1.562 \%$ \\
\hline & 0.522 & 2. & & & & $1.488 \%$ \\
\hline 0.00 & 0.495 & 2.554 & & 1.049 & $1.712 \%$ & $1.344 \%$ \\
\hline & $t i$ & & J.2 & .0 & $1.556 \%$ & 1.2 \\
\hline
\end{tabular}

Table 4: Investment strategy, optimal operational triggers and counterfactual effects. Parameter values are $\rho=0.025, \mu=0, \eta=0, c=1, S=0, R=1$, and $Q=1$ (Bar-Ilan and Strange, 1996).

although the loss in value is modest when capacity can be varied, the counterfactual effects are stronger when capacity is fixed to some arbitrary level.

\section{Conclusions}

We extend the literature on entry and exit decisions by giving the firm the opportunity to optimize the size of its capacity at the moment that it invests in the market. Capacity is assumed to be lumpy. Therefore, after each entry decision, it uses the capacity that it initially adopted. We assume that resuming operations cannot occur instantaneously because it takes time to find new employees and train them to a desired level. Besides the obvious examples of a production facility or a power plant, we can also think about a company that provides services, which needs to freeze or merge some divisions in recessions.

We find that the additional opportunity of a firm to optimize capacity changes the results to a great extent. First, a slightly higher level of uncertainty results in a delay in the optimal moment to resume operations and hastens the suspension. The latter differs from the analysis without capacity optimization, in which the firm delays the decision to suspend operations under a higher level of uncertainty. Under capacity optimization, the firm increases the size of its capacity investment, and, thus, also a higher price intercept is required for a revenue flow equal to zero. Due to positive switching costs, the firm switches from the operational state towards the suspended state for a slightly negative revenue flow, which will occur later under a higher capacity level. 
Second, we find that the length of the time lag positively affects the size of capacity. Namely, the ability of a firm to suspend operations means that the downside of the investment is truncated. Therefore, the presence of a time lag causes an increase in the expected profit over the time lag period under the assumed uncertainty. Thus, a longer time lag also positively affects the expected profit and, therefore, justifies the larger capacity choice. Under the assumption of a fixed capacity, we confirm Bar-Ilan and Strange (1996)'s result that a longer time lag results in lower switching triggers. This result is not always true under capacity optimization, however. Besides the mentioned direct effect, the triggers are also indirectly affected by an increase in the length of the time lags. A slightly longer time lag results in a higher capacity choice, thereby yielding higher switching triggers. This indirect effect dominates for an initially small time lag.

We assume an additive demand structure because this enables us to compare our results with Dangl (1999) and Hagspiel et al. (2016). However, Boonman and Hagspiel (2014) highlight the differences in results between additive and multiplicative demand structures. Where for the additive demand structure an increase in uncertainty results in a explosive increase in the capacity level, this increase is more moderate for the multiplicative demand structure. In this paper, we find that a higher level of uncertainty results in a higher trigger value to suspend the operation, which is indirectly caused by the steep increase in capacity. We leave it to further investigation to find out whether these results still hold under multiplicative demand. Additionally, it would be interesting to see how the results are affected in a competitive environment. Finally, devising capacity-investment strategies under lags and regime switches that are exogenous, e.g., that depend on technological innovation or changes in government policy (Chronopoulos and Lumbreras, 2016; Chronopoulos and Siddiqui, 2015), would also be relevant.

\section{Acknowledgements}

We are grateful to the editor and two anonymous referees for helpful suggestions that have improved the paper. All remaining errors are the authors' own.

\section{References}

Anand, K. S., Girotra, K., 2007. The strategic perils of delayed differentiation. Management Science 53 (5), 697-712.

Bar-Ilan, A., Strange, W. C., 1996. Investment lags. American Economic Review 86 (3), $610-622$. 
Boonman, H. J., Hagspiel, V., 2014. Sensitivity of demand function choice in a strategic real options context. Working paper, Tilburg University.

Boonman, H. J., Hagspiel, V., Kort, P. M., 2015. Dedicated vs. product flexible production technology: Strategic capacity investment choice. European Journal of Operational Research 244 (1), 141-152.

Brennan, M. J., Schwartz, E. S., 1985. Evaluating natural resource investments. Journal of Business 58 (2), 135-157.

Chod, J., Rudi, N., 2005. Resource flexibility with responsive pricing. Operations Research $53(3), 532-548$.

Chronopoulos, M., Lumbreras, S., 2016. Optimal regime switching under risk aversion and uncertainty. European Journal of Operational Research, forthcoming.

Chronopoulos, M., Siddiqui, A. S., 2015. When is it better to wait for a new version? Optimal replacement of an emerging technology under uncertainty. Annals of Operations Research 235 (1), 177-201.

Costeniuc, M., Schnetzer, M., Taschini, L., 2008. Entry and exit decision problem with implementation delay. Journal of Applied Probability 45(4), 1039-1059.

Dangl, T., 1999. Investment and capacity choice under uncertain demand. European Journal of Operational Research 117, 415-428.

Deneckere, R., Marvel, H. P., Peck, J., 1997. Demand uncertainty and price maintenance: markdowns as destructive competition. American Economic Review 87 (4), 619-641.

Dixit, A., 1989. Entry and exit decision under uncertainty. Journal of Political Economy $97(3), 620-638$.

Dixit, A. K., Pindyck, R. S., 1994. Investment under Uncertainty. Princeton University Press.

Etheridge, A., 2002. A Course in Financial Calculus. Cambridge University Press.

Gauthier, L., Morellec, E., 2000. Investment under uncertainty with implementation delay. In Innovation and Strategy: New Developments and Applications in Real Options (E. Schwartz and L. Trigeorgis, eds.). Oxford University Press.

Hagspiel, V., Huisman, K.J.M., Kort, P.M., 2016. Volume flexibility and capacity investment under uncertainty. International Journal of Production Economics 178, 95-108. 
McDonald, R. L., 2002. Derivatives Markets. Addison Wesley.

Mossin, J., 1968. An optimal policy for lay-up decisions. Swedish Journal of Economics 70, $170-177$.

Sødal, S., 2006. Entry and exit decisions based on a discount factor approach. Journal of Economic Dynamics and Control 30 (11), 1963-1986.

Takashima, R., Goto, M., Tsujimura, M., 2010. Irreversible investment, operating flexibility, and time lags. Asia-Pacific Journal of Operational Research 27 (2), 271-286.

Tsekrekos, A. E., 2010. The effect of mean reversion on entry and exit decisions under uncertainty. Journal of Economic Dynamics and Control 34 (4), 725-742.

van der Weijde, A. H., Hobbs, B. F., 2012. The economics of planning electricity transmission to accommodate renewables: Using two-stage optimisation to evaluate flexibility and the cost of disregarding uncertainty. Energy Economics 34 (6), 2089-2101.

Van Mieghem, J. A., Dada, M., 1999. Price versus production postponement: capacity and competition. Management Science 45 (12), 1631-1649.

\section{Appendix A. Nomenclature}

$t$

$T$

$S$

$R$

$P_{t}$

$X_{t}$

$\eta$

$Q_{t}$

$\mu$

$\sigma$

$\rho$

$\beta_{1}$

$\beta_{2}$

$c$
Time index (years)

Time lag after investment has been undertaken or production resumed (years)

Switching cost for suspending operations $(€)$

Switching cost for resuming production $(€)$

Price earned from operations at time $t(€ /$ unit)

Intercept of the inverse demand function at time $t(€ /$ unit)

Slope of the inverse demand function $\left(€ /\right.$ unit $\left.^{2}\right)$

Annual production of firm in operation at time $t$ (units)

Drift parameter of geometric Brownian motion (1/year)

Volatility parameter of geometric Brownian motion (1/year)

Annual long term interest rate (1/year)

Positive root of characteristic quadratic (-)

Negative root of characteristic quadratic (-)

Variable production cost (€/unit) 


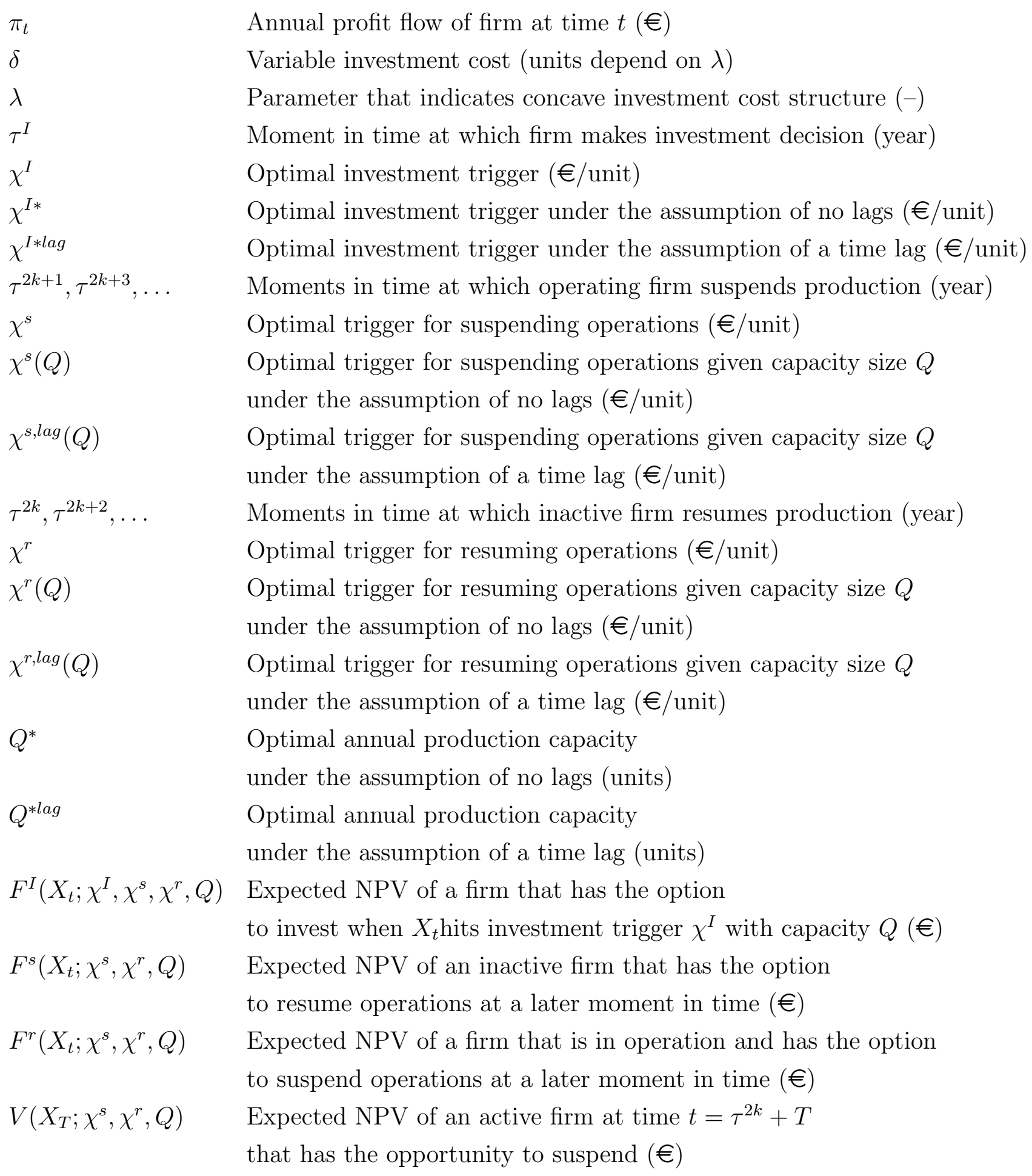

Annual profit flow of firm at time $t(€)$ Variable investment cost (units depend on $\lambda$ )

Parameter that indicates concave investment cost structure $(-)$ Moment in time at which firm makes investment decision (year) Optimal investment trigger (€/unit) Optimal investment trigger under the assumption of no lags (€/unit) Optimal investment trigger under the assumption of a time lag (€/unit) Moments in time at which operating firm suspends production (year) Optimal trigger for suspending operations (€/unit) Optimal trigger for suspending operations given capacity size $Q$ under the assumption of no lags ( $€$ /unit) Optimal trigger for suspending operations given capacity size $Q$ under the assumption of a time lag (€/unit) Moments in time at which inactive firm resumes production (year) Optimal trigger for resuming operations (€/unit) Optimal trigger for resuming operations given capacity size $Q$ under the assumption of no lags (€/unit)

Optimal trigger for resuming operations given capacity size $Q$ under the assumption of a time lag (€/unit)

Optimal annual production capacity under the assumption of no lags (units) Optimal annual production capacity under the assumption of a time lag (units) Expected NPV of a firm that has the option to invest when $X_{t}$ hits investment trigger $\chi^{I}$ with capacity $Q(€)$ Expected NPV of an inactive firm that has the option to resume operations at a later moment in time $(€)$ Expected NPV of a firm that is in operation and has the option to suspend operations at a later moment in time $(€)$ Expected NPV of an active firm at time $t=\tau^{2 k}+T$ that has the opportunity to suspend (€)

\section{Appendix B. Expected Value of the Option to Suspend}

When parameter $X_{T}$ follows a GBM, the distribution of $X_{T}$ given $\chi^{r}$ is $\log$ normal, i.e.

$$
\left.\log \left(X_{T}\right)\right|_{\chi^{r}} \sim N\left(\log \left(\chi^{r}\right)+\left(\mu-\frac{1}{2} \sigma^{2}\right) T, \sigma^{2} T\right)
$$


Let us assume that $z=\log \left(X_{T}\right), g=\log \left(\chi^{r}\right)+\left(\mu-\frac{1}{2} \sigma^{2}\right) T$ and $s^{2}=\sigma^{2} T$, then

$$
z \sim N\left(g, s^{2}\right)
$$

We are interested in $\mathbb{E}\left[X_{T}^{\beta} \mid \chi^{r}\right]=\mathbb{E}\left[e^{z \beta} \mid \chi^{r}\right]$. This is in fact equal to the calculation of the moment generating function. However, when we only need this expectation for $X_{T} \in\left(0, \chi^{s}\right)$, then the $\beta$ th moment of $X_{T}$ is calculated as follows:

$$
\mathbb{E}\left[e^{z \beta} \mid z<\log \left(\chi^{s}\right)\right]=\int_{-\infty}^{\log \left(\chi^{s}\right)} e^{z \beta} \frac{1}{\sqrt{2 \pi s^{2}}} e^{-\frac{1}{2} \frac{(z-g)^{2}}{s^{2}}} d z
$$

Define $y=\frac{z-g}{s}$, which implies that $z=y s+g$. The substitution rule gives

$$
\mathbb{E}\left[e^{z \beta} \mid z<\log \left(\chi^{s}\right)\right]=\int_{-\infty}^{\frac{\log \left(\chi^{s}\right)-g}{s}} e^{(y s+g) \beta} \frac{1}{\sqrt{2 \pi s^{2}}} e^{-\frac{1}{2} y^{2}}\left(\frac{d z}{d y}\right) d y
$$

Because we know that $\frac{d z}{d y}=s$, we find:

$$
\mathbb{E}\left[e^{z \beta} \mid z<\log \left(\chi^{s}\right)\right]=e^{\beta g} \int_{-\infty}^{\frac{\log \left(\chi^{s}\right)-g}{s}} e^{y s \beta} \frac{1}{\sqrt{2 \pi}} e^{-\frac{1}{2} y^{2}} d y
$$

Replacement of some terms yields:

$$
\begin{aligned}
& \mathbb{E}\left[e^{z \beta} \mid z<\log \left(\chi^{s}\right)\right]=e^{\beta g} \int_{-\infty}^{\frac{\log \left(\chi^{s}\right)-g}{s}} \frac{1}{\sqrt{2 \pi}} e^{-\frac{1}{2}(y-\beta s)^{2}} e^{\frac{1}{2} s^{2} \beta^{2}} d y, \\
& \mathbb{E}\left[e^{z \beta} \mid z<\log \left(\chi^{s}\right)\right]=e^{\beta g+\frac{1}{2} s^{2} \beta^{2}} \int_{-\infty}^{\frac{\log \left(\chi^{s}\right)-g}{s}} \frac{1}{\sqrt{2 \pi}} e^{-\frac{1}{2}(y-\beta s)^{2}} d y .
\end{aligned}
$$

Finally, the integral is defined as a CDF of variable $y$ that is normally distributed with expectation $\beta s$ and variance 1 . When we also substitute $y=\frac{z-g}{s}$ back in the integral, we find:

$$
\begin{aligned}
\mathbb{E}\left[e^{z \beta} \mid z<\log \left(\chi^{s}\right)\right] & =\mathbb{E}\left[X_{T}^{\beta} \mid X_{T}<\chi^{s}\right] \\
& =e^{\beta g+\frac{1}{2} s^{2} \beta^{2}} \Phi\left(\frac{\log \left(\chi^{s}\right)-g}{s}-\beta s\right)
\end{aligned}
$$

thus

$$
\mathbb{E}\left[e^{z \beta} \mid z<\log \left(\chi^{s}\right)\right]=\left(\chi^{r}\right)^{\beta} e^{\frac{1}{2} \sigma^{2} \beta^{2} T+\left(\mu-\frac{1}{2} \sigma^{2}\right) \beta T} \Phi\left(\frac{\log \left(\chi^{s}\right)-\log \left(\chi^{r}\right)-\left(\mu-\frac{1}{2} \sigma^{2} T\right)}{\sigma \sqrt{T}}-\beta \sigma \sqrt{T}\right) .
$$


Since $\beta_{1}\left(\beta_{2}\right)$ is the positive (negative) root of the quadratic polynomial

$$
\frac{1}{2} \sigma^{2} \beta^{2}+\left(\mu-\frac{1}{2} \sigma^{2}\right) \beta-\rho=0,
$$

we find that $e^{\frac{1}{2} \sigma^{2} \beta^{2} T+\left(\mu-\frac{1}{2} \sigma^{2}\right) \beta T}=e^{\rho T}$. By applying this result to the components of the integral in expression (12), we obtain:

$$
\begin{aligned}
& \Rightarrow \int_{0}^{\chi^{s}}\left(\frac{X_{T}}{\chi^{r}}\right)^{\beta_{1}}\left(F^{r}\left(\chi^{r} ; \chi^{s}, \chi^{r}, Q\right)\right) f\left(X_{T} \mid \chi^{r}\right) d X_{T} \\
& =\left(\frac{\chi^{r}}{\chi^{r}}\right)^{\beta_{1}}\left(F^{r}\left(\chi^{r} ; \chi^{s}, \chi^{r}, Q\right)\right) \Phi\left(v\left(\chi^{s}, \chi^{r}\right)-\beta_{1} \sigma \sqrt{T}\right) e^{\rho T} \\
& \Rightarrow-\int_{0}^{\chi^{s}}(R+S) f\left(X_{T} \mid \chi^{r}\right) d X_{T}=-(S+R) \Phi\left(v\left(\chi^{s}, \chi^{r}\right)\right) \\
& \Rightarrow \int_{\chi^{s}}^{\infty} \frac{X_{T} Q}{\rho-\mu} f\left(X_{T} \mid \chi^{r}\right) d X_{T}=\frac{\chi^{r} Q}{\rho-\mu}\left(1-\Phi\left(v\left(\chi^{s}, \chi^{r}\right)-\sigma \sqrt{T}\right)\right) e^{\mu T} \\
& \Rightarrow-\int_{\chi^{s}}^{\infty}\left(\frac{\left(\eta Q^{2}+c Q\right)}{\rho}+R\right) f\left(X_{T} \mid \chi^{r}\right) d X_{T} \\
& =-\left(\frac{\left(\eta Q^{2}+c Q\right)}{\rho}+R\right)\left(1-\Phi\left(v\left(\chi^{s}, \chi^{r}\right)\right)\right) \\
& \Rightarrow \int_{\chi^{s}}^{\infty}\left(\frac{X_{T}}{\chi^{s}}\right)^{\beta_{2}}\left(\frac{\left(\eta Q^{2}+c Q\right)}{\rho}-\frac{\chi^{s} Q}{\rho-\mu}-S+\left(\frac{\chi^{s}}{\chi^{r}}\right)^{\beta_{1}} F^{r}\left(\chi^{r} ; \chi^{s}, \chi^{r}, Q\right)\right) f\left(X_{T} \mid \chi^{r}\right) d X_{T} \\
& =\left(\frac{\chi^{r}}{\chi^{s}}\right)^{\beta_{2}}\left(\frac{\left(\eta Q^{2}+c Q\right)}{\rho}-\frac{\chi^{s} Q}{\rho-\mu}-S+\left(\frac{\chi^{s}}{\chi^{r}}\right)^{\beta_{1}} F^{r}\left(\chi^{r} ; \chi^{s}, \chi^{r}, Q\right)\right) \\
& \cdot\left(1-\Phi\left(v\left(\chi^{s}, \chi^{r}\right)-\beta_{2} \sigma \sqrt{T}\right)\right) e^{\rho T}
\end{aligned}
$$




\section{Appendix C. Proof of Proposition 1}

We show that expression (11) moves towards expression (4) for $T \rightarrow 0$. For this, we introduce new notation to distinguish the expected NPVs for a model with a time lag from the expected NPVs without a time lag. $F^{r}\left(\chi^{r}, T\right)$ denotes the expected NPV of a firm that has just made the decision to resume production and now obtains the option to suspend at an optimal moment after the time lag. For the model without a time lag, we define this option by $F^{r}\left(\chi^{r}, 0\right)$. We will show that $\lim _{T \rightarrow 0} F^{r}\left(\chi^{r}, T\right)=F^{r}\left(\chi^{r}, 0\right)$. Substituting (13) into (11) gives:

$$
\begin{aligned}
& F^{r}\left(\chi^{r}, T\right) \\
= & e^{-\rho T}\left(\left(\frac{\chi^{r}}{\chi^{r}}\right)^{\beta_{1}} F^{r}\left(\chi^{r}, T\right) \Phi\left(v\left(\chi^{s}, \chi^{r}\right)-\beta_{1} \sigma \sqrt{T}\right) e^{\rho T}\right. \\
+ & \frac{\chi^{r} Q}{\rho-\mu}\left(1-\Phi\left(v\left(\chi^{s}, \chi^{r}\right)-\sigma \sqrt{T}\right)\right) e^{\mu T}-\left(\frac{\left(\eta Q^{2}+c Q\right)}{\rho}+R\right)\left(1-\Phi\left(v\left(\chi^{s}, \chi^{r}\right)\right)\right) \\
- & (S+R) \Phi\left(v\left(\chi^{s}, \chi^{r}\right)\right)+\left(\frac{\chi^{r}}{\chi^{s}}\right)^{\beta_{2}}\left(\frac{\left(\eta Q^{2}+c Q\right)}{\rho}-\frac{\chi^{s} Q}{\rho-\mu}-S\right. \\
+ & \left.\left.\left(\frac{\chi^{s}}{\chi^{r}}\right)^{\beta_{1}} F^{r}\left(\chi^{r}, T\right)\right)\left(1-\Phi\left(v\left(\chi^{s}, \chi^{r}\right)-\beta_{2} \sigma \sqrt{T}\right)\right) e^{\rho T}\right) .
\end{aligned}
$$

Since $\chi^{r} \geq \chi^{s}$, we find that $v\left(\chi^{s}, \chi^{r}\right)<0$, where $v\left(\chi^{s}, \chi^{r}\right)$ is defined by expression (14). For $T \rightarrow 0$, it holds that $v\left(\chi^{s}, \chi^{r}\right) \rightarrow-\infty$. Thus, $\lim _{T \rightarrow 0} \Phi\left(v\left(\chi^{s}, \chi^{r}\right)\right)=0$ and $\lim _{T \rightarrow 0} \Phi\left(v\left(\chi^{s}, \chi^{r}\right)-\right.$ $\left.\beta_{1} \sigma \sqrt{T}\right)=0$, which eliminates two terms in expression (C-1). Similarly, we obtain $\lim _{T \rightarrow 0}$ $\left(1-\Phi\left(v\left(\chi^{s}, \chi^{r}\right)-\sigma \sqrt{T}\right)\right)=1, \lim _{T \rightarrow 0}\left(1-\Phi\left(v\left(\chi^{s}, \chi^{r}\right)\right)\right)=1$, and $\lim _{T \rightarrow 0}\left(1-\Phi\left(v\left(\chi^{s}, \chi^{r}\right)-\beta_{2} \sigma \sqrt{T}\right)\right)$ $=1$. In order to finish the proof, we additionally have $\lim _{T \rightarrow 0} e^{\rho T}=1$ and $\lim _{T \rightarrow 0} e^{\mu T}=1$. Hence, we find that:

$$
\begin{aligned}
F^{r}\left(\chi^{r}, 0\right)=\frac{\chi^{r} Q}{\rho-\mu}- & \frac{\left(\eta Q^{2}+c Q\right)}{\rho}-R \\
& +\left(\frac{\chi^{r}}{\chi^{s}}\right)^{\beta_{2}}\left(\frac{\left(\eta Q^{2}+c Q\right)}{\rho}-\frac{\chi^{s} Q}{\rho-\mu}-S+\left(\frac{\chi^{s}}{\chi^{r}}\right)^{\beta_{1}} F^{r}\left(\chi^{r}, 0\right)\right),
\end{aligned}
$$

which is similar to expression (4) with $X=\chi^{r}$, after substitution of expression (6) in which $X_{t}=\chi^{s}$. Notice that, due to the assumption that switching cost, $R$, is paid after the time lag, $R$ is placed in a different position in the above formula than in expression (4). However, since $T \rightarrow 0$, this does not affect the results. 